\title{
Matching theorems for systems of a finitely generated Coxeter group
}

\author{
Michael L MiHAliK \\ JOHN G RATCLIFFE \\ STEVEn T TSCHANTZ
}

\begin{abstract}
We study the relationship between two sets $S$ and $S^{\prime}$ of Coxeter generators of a finitely generated Coxeter group $W$ by proving a series of theorems that identify common features of $S$ and $S^{\prime}$. We describe an algorithm for constructing from any set of Coxeter generators $S$ of $W$ a set of Coxeter generators $R$ of maximum rank for $W$.

A subset $C$ of $S$ is called complete if any two elements of $C$ generate a finite group. We prove that if $S$ and $S^{\prime}$ have maximum rank, then there is a bijection between the complete subsets of $S$ and the complete subsets of $S^{\prime}$ so that corresponding subsets generate isomorphic Coxeter systems. In particular, the Coxeter matrices of $(W, S)$ and $\left(W, S^{\prime}\right)$ have the same multiset of entries.
\end{abstract}

\section{Introduction}

The isomorphism problem for finitely generated Coxeter groups is the problem of deciding if two finite Coxeter matrices define isomorphic Coxeter groups. Coxeter [5] solved this problem for finite irreducible Coxeter groups. Recently there has been considerable interest and activity on the isomorphism problem for arbitrary finitely generated Coxeter groups. For a recent survey, see Mühlherr [11].

The isomorphism problem for finitely generated Coxeter groups is equivalent to the problem of determining all the automorphism equivalence classes of sets of Coxeter generators for an arbitrary finitely generated Coxeter group. In this paper, we study the relationship between two sets $S$ and $S^{\prime}$ of Coxeter generators of a finitely generated Coxeter group $W$ by proving a series of matching theorems that identify common features of $S$ and $S^{\prime}$.

A basic subset of $S$ is a maximal subset $B$ of $S$ such that $B$ generates an irreducible, noncyclic, finite subgroup of $W$. The point of departure of our paper is the following matching theorem (Theorem 4.18). 
Basic Matching Theorem Let $W$ be a finitely generated Coxeter group with two sets of Coxeter generators $S$ and $S^{\prime}$. Then there is a natural bijection (matching) between the basic subsets of $S$ and the basic subsets of $S^{\prime}$.

A basic subset $B$ of $S$ matches a basic subset $B^{\prime}$ of $S^{\prime}$ if and only if $[\langle B\rangle,\langle B\rangle]$ is conjugate to $\left[\left\langle B^{\prime}\right\rangle,\left\langle B^{\prime}\right\rangle\right]$ in $W$. Usually matching basic subsets generate isomorphic groups, in which case, we say that the basic subsets match isomorphically; however, there are exceptions, due to well known isomorphisms between irreducible and reducible finite Coxeter groups (for instance the dihedral group $\mathbf{D}_{2}$ (6) of order 12 and $\mathbf{A}_{1} \times \mathbf{A}_{2}$ ). We show that nonisomorphic matching of basic subsets can be understood by blowing up Coxeter generating sets. This is a procedure to replace a given Coxeter generating set $S$ by a Coxeter generating set $R$ such that $|R|=|S|+1$. We prove that if two Coxeter generating sets $S$ and $S^{\prime}$ for $W$ have nonisomorphically matching basic subsets, then either $S$ or $S^{\prime}$ can be blown up.

We describe a simple algorithm to determine if $S$ can be blown up. We prove that every sequence $S_{1}, S_{2}, \ldots$ of Coxeter generators of $W$, such that $S_{i+1}$ is obtained from $S_{i}$ by blowing up $S_{i}$ for each $i$, terminates in a set of Coxeter generators $R$ that has maximum rank $|R|$ over all sets of Coxeter generators of $W$. If $R$ and $R^{\prime}$ are sets of Coxeter generators of $W$ of maximum rank, then the basic subsets of $R$ and $R^{\prime}$ match isomorphically, since neither $R$ nor $R^{\prime}$ can be blown up.

A subset $C$ of $S$ is called complete if any two elements of $C$ generate a finite group. The main result of our paper is the following matching theorem (Theorem 7.7).

Simplex Matching Theorem Let $W$ be a finitely generated Coxeter group with two sets of Coxeter generators $S$ and $S^{\prime}$ whose basic subsets match isomorphically. Then there is a bijection between the complete subsets of $S$ and the complete subsets of $S^{\prime}$ so that corresponding subsets generate isomorphic Coxeter systems. In particular, $|S|=\left|S^{\prime}\right|$, and the Coxeter matrices of $(W, S)$ and $\left(W, S^{\prime}\right)$ have the same multiset of entries.

\section{Acknowledgements}

The authors thank the referee for the suggestions that greatly improved the exposition of this paper.

\section{Preliminaries}

A Coxeter matrix is a symmetric matrix $M=(m(s, t))_{s, t \in S}$ with $m(s, t)$ either a positive integer or infinity and $m(s, t)=1$ if and only if $s=t$. A Coxeter system with 
Coxeter matrix $M=(m(s, t))_{s, t \in S}$ is a pair $(W, S)$ consisting of a group $W$ and a set of generators $S$ for $W$ such that $W$ has the presentation

$$
\left.W=\langle S|(s t)^{m(s, t)}: s, t \in S \text { and } m(s, t)<\infty\right\rangle .
$$

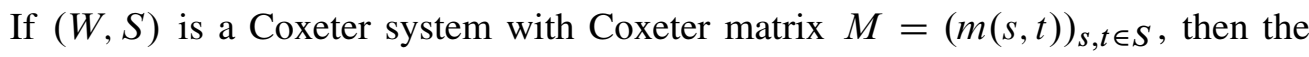
order of $s t$ is $m(s, t)$ for each $s, t$ in $S$ by Bourbaki [1, Proposition 4], and so a Coxeter system $(W, S)$ determines its Coxeter matrix; moreover, any Coxeter matrix $M=(m(s, t))_{s, t \in S}$ determines a Coxeter system $(W, S)$ where $W$ is defined by the above presentation. If $(W, S)$ is a Coxeter system, then $W$ is called a Coxeter group and $S$ is called a set of Coxeter generators for $W$, and the cardinality of $S$ is called the rank of $(W, S)$. A Coxeter system $(W, S)$ has finite rank if and only if $W$ is finitely generated by [1, Theorem 2(iii)].

Let $(W, S)$ be a Coxeter system. A visible subgroup of $(W, S)$ is a subgroup of $W$ of the form $\langle A\rangle$ for some $A \subseteq S$. A visible subgroup is also called a special subgroup or a standard parabolic subgroup. If $\langle A\rangle$ is a visible subgroup of $(W, S)$, then $(\langle A\rangle, A)$ is also a Coxeter system by [1, Theorem 2(i)].

When studying a Coxeter system $(W, S)$ with Coxeter matrix $M$ it is helpful to have a visual representation of $(W, S)$. There are two graphical ways of representing $(W, S)$ and we shall use both depending on our needs.

The Coxeter diagram (C-diagram) of $(W, S)$ is the labeled undirected graph $\Delta(W, S)$ with vertices $S$ and edges

$$
\{(s, t): s, t \in S \text { and } m(s, t)>2\}
$$

such that an edge $(s, t)$ is labeled by $m(s, t)$. Coxeter diagrams are useful for visually representing finite Coxeter groups. If $A \subset S$, then $\Delta(\langle A\rangle, A)$ is the subdiagram of $\Delta(W, S)$ induced by $A$.

A Coxeter system $(W, S)$ is said to be irreducible if its $\mathrm{C}$-diagram is connected. A visible subgroup $\langle A\rangle$ of $(W, S)$ is said to be irreducible if $(\langle A\rangle, A)$ is irreducible. A subset $A$ of $S$ is said to be irreducible if $\langle A\rangle$ is irreducible.

A subset $A$ of $S$ is said to be a component of $S$ if $A$ is a maximal irreducible subset of $S$ or equivalently if $\Delta(\langle A\rangle, A)$ is a connected component of $\Delta(W, S)$. The connected components of $\Delta(W, S)$ represent the factors of a direct product decomposition of $W$.

The presentation diagram (P-diagram) of $(W, S)$ is the labeled undirected graph $\Gamma(W, S)$ with vertices $S$ and edges

$$
\{(s, t): s, t \in S \text { and } m(s, t)<\infty\}
$$

Algebraic $8 \mathcal{G}$ Geometric Topology, Volume 7 (2007) 
such that an edge $(s, t)$ is labeled by $m(s, t)$. Presentation diagrams are useful for visually representing infinite Coxeter groups. If $A \subset S$, then $\Gamma(\langle A\rangle, A)$ is the subdiagram of $\Gamma(W, S)$ induced by $A$. The connected components of $\Gamma(W, S)$ represent the factors of a free product decomposition of $W$.

Example Consider the Coxeter group $W$ generated by the four reflections in the sides of a rectangle in $E^{2}$. The C-diagram of $(W, S)$ is the disjoint union of two edges labeled by $\infty$.

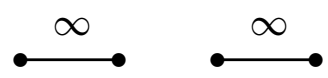

Therefore $W$ is the direct product of two infinite dihedral groups. The $\mathrm{P}$-diagram of $W$ is a square with edge labels 2 .

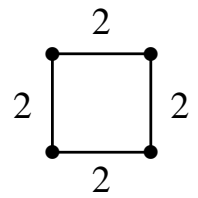

Let $(W, S)$ and $\left(W^{\prime}, S^{\prime}\right)$ be Coxeter systems with P-diagrams $\Gamma$ and $\Gamma^{\prime}$, respectively. An isomorphism $\phi:(W, S) \rightarrow\left(W^{\prime}, S^{\prime}\right)$ of Coxeter systems is an isomorphism $\phi: W \rightarrow$ $W^{\prime}$ such that $\phi(S)=S^{\prime}$. An isomorphism $\psi: \Gamma \rightarrow \Gamma^{\prime}$ of P-diagrams is a bijection from $S$ to $S^{\prime}$ that preserves edges and their labels. Clearly, two Coxeter systems are isomorphic if and only if their $\mathrm{P}$-diagrams are isomorphic.

A Coxeter group $W$ is said to be rigid if for any two sets of Coxeter generators $S$ and $S^{\prime}$ for $W$, there is an isomorphism $\alpha:(W, S) \rightarrow\left(W, S^{\prime}\right)$ or equivalently any two sets of Coxeter generators $S$ and $S^{\prime}$ for $W$ determine isomorphic P-diagrams for $W$. A Coxeter group $W$ is said to be strongly rigid if any two sets of Coxeter generators for $W$ are conjugate.

A Coxeter system $(W, S)$ is said to be complete if the underlying graph of the P-diagram of $(W, S)$ is complete. A complete Coxeter system is also called a 2-spherical Coxeter system. A Coxeter system $(W, S)$ is said to be finite (resp. infinite) if $W$ is finite (resp. infinite).

Theorem 2.1 Caprace, Franzsen, Howlett, and Mühlherr [4;9] If $(W, S)$ is an infinite, complete, irreducible Coxeter system of finite rank, then $W$ is strongly rigid.

\section{Coxeter systems of finite Coxeter groups}

We shall use Coxeter's notation [6, page 297] for the irreducible spherical Coxeter simplex reflection groups except that we denote the dihedral group $\mathbf{D}_{2}^{k}$ by $\mathbf{D}_{2}(k)$. 
Subscripts denote the rank of a Coxeter system in Coxeter's notation. Coxeter's notation partly agrees with but differs from Bourbaki's notation [1, page 193].

Coxeter [5] proved that every finite irreducible Coxeter system is isomorphic to exactly one of the Coxeter systems $\mathbf{A}_{n}, n \geq 1, \mathbf{B}_{n}, n \geq 4, \mathbf{C}_{n}, n \geq 2, \mathbf{D}_{2}(k), k \geq 5, \mathbf{E}_{6}$, $\mathbf{E}_{7}, \mathbf{E}_{8}, \mathbf{F}_{4}, \mathbf{G}_{3}, \mathbf{G}_{4}$. Each of these Coxeter groups, of rank $n$, is a finite group of orthogonal $n \times n$ matrices. The center of each of these Coxeter groups is either $\{I\}$ or $\{ \pm I\}$ and the groups with center $\{I\}$ are $\mathbf{A}_{n}, n>1, \mathbf{B}_{n}, n$ odd, $\mathbf{D}_{2}(k), k$ odd, and $\mathbf{E}_{6}$. We denote the center of a group $G$ by $Z(G)$. If $G$ is a group of orthogonal matrices, we denote the subgroup of determinant 1 matrices in $G$ by $G^{+}$.

The type of a finite irreducible Coxeter system $(W, S)$ is the isomorphism type of $(W, S)$ represented by one of the systems $\mathbf{A}_{n}, \mathbf{B}_{n}, \mathbf{C}_{n}, \mathbf{D}_{2}(k), \mathbf{E}_{6}, \mathbf{E}_{7}, \mathbf{E}_{8}, \mathbf{F}_{4}, \mathbf{G}_{3}$, $\mathbf{G}_{4}$. The type of an irreducible subset $A$ of $S$ is the type of $(\langle A\rangle, A)$.

The Coxeter group $\mathbf{A}_{n}$ is the group of symmetries of a regular $n$-simplex for each $n \geq 1$, and so $\mathbf{A}_{n}$ is isomorphic to the symmetric group $S_{n+1}$ for each $n \geq 1$. The C-diagram of $\mathbf{A}_{n}$ is a linear diagram with $n$ vertices and all edge labels 3. The Coxeter generators $a_{1}, \ldots, a_{n}$ of $\mathbf{A}_{n}$, indexed so that $m\left(a_{i}, a_{i+1}\right)=3$ for $i=1, \ldots, n-1$, correspond to the transpositions (12), (23), ., $(n n+1)$ of $S_{n+1}$.

The Coxeter group $\mathbf{C}_{n}$ is the group of symmetries of an $n$-cube for each $n \geq 2$, and $\mathbf{C}_{n}$ is represented by the group of all $n \times n$ orthogonal matrices in which each column has all zero entries except for one, which is \pm 1 . The $\mathrm{C}$-diagram of $\mathbf{C}_{n}$ is a linear diagram with $n$ vertices and all edge labels 3 except for the last edge labelled 4. The Coxeter generators $c_{1}, \ldots, c_{n}$ of $\mathbf{C}_{n}$ are indexed so that $m\left(c_{i}, c_{i+1}\right)=3$ for $i=1, \ldots, n-2$ and $m\left(c_{n-1}, c_{n}\right)=4$. The generators $c_{1}, \ldots, c_{n-1}$ are represented by the permutation matrices corresponding to the transpositions $(12),(23), \ldots,(n-1 n)$ and $c_{n}$ is represented by the matrix $\operatorname{diag}(1, \ldots, 1,-1)$.

The Coxeter group $\mathbf{B}_{n}$, with $n \geq 4$, is a subgroup of $\mathbf{C}_{n}$ of index 2 with Coxeter generators $b_{i}=c_{i}$, for $i=1, \ldots, n-1$, and $b_{n}=c_{n} c_{n-1} c_{n}$. We have $b_{n-1} b_{n}=$ $\operatorname{diag}(1, \ldots, 1,-1,-1)$ and $m\left(b_{n-2}, b_{n}\right)=3$. The $\mathrm{C}$-diagram of $\mathbf{B}_{n}$ is a Y-shaped diagram with $n$ vertices and all edge labels 3 and two short arms of consisting of single edges with endpoint $b_{n-1}$ and $b_{n}$. In order to have uniformity of notation, we define $\mathbf{B}_{3}=\mathbf{A}_{3}$.

The group $\mathbf{D}_{2}(k)$ is the group of symmetries of a regular $k$-gon for each $k \geq 5$. In order to have uniformity of notation, we define $\mathbf{D}_{2}(3)=\mathbf{A}_{2}$ and $\mathbf{D}_{2}(4)=\mathbf{C}_{2}$. Then $\mathbf{D}_{2}(k)$ is a dihedral group of order $2 k$ for all $k \geq 3$. The $\mathbf{C}$-diagram of $\mathbf{D}_{2}(k)$ is an edge with label $k$.

The C-diagrams of $\mathbf{E}_{6}, \mathbf{E}_{7}, \mathbf{E}_{8}$ are star shaped with three arms and all edge labels 3. One arm has length one and another arm has length two. The $\mathrm{C}$-diagram of $\mathbf{F}_{4}$ is a 
linear diagram with edge labels $3,4,3$ in that order. The $\mathbf{C}$-diagram of $\mathbf{G}_{3}$ is a linear diagram with edge labels 3,5 . The $\mathbf{C}$-diagram of $\mathbf{G}_{4}$ is a linear diagram with edge labels $3,3,5$ in that order.

Lemmas 3.1 through 3.4 are either elementary or well known.

Lemma 3.1 The Coxeter groups $\mathbf{A}_{n}, n \geq 1, \mathbf{B}_{n}, n \geq 3, \mathbf{E}_{6}, \mathbf{E}_{8}, \mathbf{F}_{4}$, and $\mathbf{G}_{4}$ are indecomposable with respect to direct products.

Lemma 3.2 The Coxeter group $\mathbf{C}_{n}$ is decomposable with respect to direct products if and only if $n$ is odd. If $n$ is odd and $\mathbf{C}_{n}=H \times K$ with $1<|H| \leq|K|$, then $H=\{ \pm I\}$ and $K=\mathbf{B}_{n}$ or $\theta\left(\mathbf{B}_{n}\right)$ where $\theta$ is the automorphism of $\mathbf{C}_{n}$ defined by $\theta\left(c_{i}\right)=-c_{i}$, for $i=1, \ldots, n-1$, and $\theta\left(c_{n}\right)=c_{n}$.

Lemma 3.3 The Coxeter group $\mathbf{D}_{2}(n)$, with Coxeter generators $a$ and $b$, is decomposable with respect to direct products if and only if $n \equiv 2 \bmod 4$. If $n \equiv 2 \bmod 4$ and $\mathbf{D}_{2}(n)=H \times K$ with $1<|H| \leq|K|$, then $H=\left\langle(a b)^{n / 2}\right\rangle$ and $K=\langle a, b a b\rangle$ or $\langle b, a b a\rangle$, moreover $K \cong \mathbf{D}_{2}(n / 2)$.

Lemma 3.4 The Coxeter groups $G=\mathbf{E}_{7}, \mathbf{G}_{3}$ are decomposable with respect to direct products. If $G=H \times K$ with $1<|H| \leq|K|$, then $H=\{ \pm I\}$ and $K=G^{+}$, moreover $G^{+}$is a nonabelian simple group.

The next lemma follows from the Krull-Remak-Schmidt Theorem (KRS Theorem), see Suzuki [14, Theorem 4.8].

Lemma 3.5 Let $G$ be a finite group with direct product decompositions

$$
G=H_{1} \times H_{2} \times \cdots \times H_{r} \text { and } G=K_{1} \times K_{2} \times \cdots \times K_{s}
$$

such that $H_{i}$ and $K_{j}$ are nontrivial and indecomposable with respect to direct products for each $i$ and $j$. Let $\iota_{i}: H_{i} \rightarrow G$ be the inclusion map for each $i$ and let $\pi_{j}: G \rightarrow K_{j}$ be the projection map for each $j$. Suppose $H_{p}$ is nonabelian. Then there is a unique $q$ such that $H_{p} \cap K_{q} \neq\{1\}$. Moreover $\pi_{q} \iota_{p}: H_{p} \rightarrow K_{q}$ is an isomorphism and $Z(G) H_{p}=Z(G) K_{q}$. Furthermore $\left[H_{p}, H_{p}\right]=\left[K_{q}, K_{q}\right]$ and $\pi_{q} \iota_{p}: H_{p} \rightarrow K_{q}$ restricts to the identity on $\left[H_{p}, H_{p}\right]$.

Theorem 3.6 (Matching Theorem for systems of a finite Coxeter group) Let $W$ be a finite Coxeter group with two sets of Coxeter generators $S$ and $S^{\prime}$. Let

$$
(W, S)=\left(W_{1}, S_{1}\right) \times \cdots \times\left(W_{m}, S_{m}\right)
$$


and

$$
\left(W, S^{\prime}\right)=\left(W_{1}^{\prime}, S_{1}^{\prime}\right) \times \cdots \times\left(W_{n}^{\prime}, S_{n}^{\prime}\right)
$$

be the factorizations of $(W, S)$ and $\left(W, S^{\prime}\right)$ into irreducible factors. Let $k$ be such that $W_{k}$ is noncyclic. Then there is a unique $\ell$ such that $W_{\ell}^{\prime}$ is noncyclic and $\left[W_{k}, W_{k}\right]=$ $\left[W_{\ell}^{\prime}, W_{\ell}^{\prime}\right]$. Moreover,

(1) $Z(W) W_{k}=Z(W) W_{\ell}^{\prime}$,

(2) if $\left|W_{k}\right|=\left|W_{\ell}^{\prime}\right|$, then $\left(W_{k}, S_{k}\right) \cong\left(W_{\ell}^{\prime}, S_{\ell}^{\prime}\right)$ and there is an isomorphism $\phi: W_{k} \rightarrow$ $W_{\ell}^{\prime}$ that restricts to the identity on $\left[W_{k}, W_{k}\right]$,

(3) if $\left|W_{k}\right|<\left|W_{\ell}^{\prime}\right|$, then either $\left(W_{k}, S_{k}\right)$ has type $\mathbf{B}_{2 q+1}$ and $\left(W_{\ell}^{\prime}, S_{\ell}^{\prime}\right)$ has type $\mathbf{C}_{2 q+1}$ for some $q \geq 1$ or $\left(W_{k}, S_{k}\right)$ has type $\mathbf{D}_{2}(2 q+1)$ and $\left(W_{\ell}^{\prime}, S_{\ell}^{\prime}\right)$ has type $\mathbf{D}_{2}(4 q+2)$ for some $q \geq 1$, and there is a monomorphism $\phi: W_{k} \rightarrow W_{\ell}^{\prime}$ that restricts to the identity on $\left[W_{k}, W_{k}\right]$.

Proof By Lemmas 3.1-3.4, we can refine the decomposition $W=W_{1} \times \cdots \times W_{m}$ to a decomposition $W=H_{1} \times \cdots \times H_{r}$, with $H_{i}$ nontrivial and indecomposable with respect to direct products, by replacing each $W_{i}$ that factors into a direct product $W_{i}=H_{j-1} \times H_{j}$, with $\left|H_{j-1}\right|=2$, by $H_{j-1} \times H_{j}$. Likewise refine the decomposition $W=W_{1}^{\prime} \times \cdots \times W_{\ell}^{\prime}$ to a decomposition $W=K_{1} \times \cdots \times K_{s}$, with $K_{i}$ nontrivial and indecomposable with respect to direct products, by replacing each $W_{i}^{\prime}$ that factors into a direct product $W_{i}^{\prime}=K_{j-1} \times K_{j}$, with $\left|K_{j-1}\right|=2$, by $K_{j-1} \times K_{j}$. Then $r=s$ by the KRS Theorem.

Suppose that $W_{k}$ is noncyclic. Then $W_{k}$ is nonabelian, since $\left(W_{k}, S_{k}\right)$ is irreducible. Now $W_{k}=H_{p}$ or $H_{p-1} \times H_{p}$, with $\left|H_{p-1}\right|=2$, for some $p$. In either case $H_{p}$ is nonabelian by Lemmas 3.1-3.4. By Lemma 3.5, there is a unique $q$ such that $H_{p} \cap K_{q} \neq\{1\}$. Moreover $\left[H_{p}, H_{p}\right]=\left[K_{q}, K_{q}\right]$ and $\pi_{q} \iota_{p}: H_{p} \rightarrow K_{q}$ restricts to the identity on $\left[H_{p}, H_{p}\right]$. Then $K_{q}$ is nonabelian. Now there is an $\ell$ such that $W_{\ell}^{\prime}=K_{q}$ or $K_{q-1} \times K_{q}$ with $\left|K_{q-1}\right|=2$. Then $W_{\ell}^{\prime}$ is noncyclic and

$$
\left[W_{k}, W_{k}\right]=\left[H_{p}, H_{p}\right]=\left[K_{q}, K_{q}\right]=\left[W_{\ell}^{\prime}, W_{\ell}^{\prime}\right]
$$

Now suppose $W_{i}^{\prime}$ is noncyclic and $\left[W_{k}, W_{k}\right]=\left[W_{i}^{\prime}, W_{i}^{\prime}\right]$. Then $W_{i}^{\prime}$ is nonabelian, since $W_{k}$ is nonabelian. Now $W_{i}^{\prime}=K_{j}$ or $K_{j-1} \times K_{j}$, with $\left|K_{j-1}\right|=2$, for some $j$. Then we have

$$
\left[H_{p}, H_{p}\right]=\left[W_{k}, W_{k}\right]=\left[W_{i}^{\prime}, W_{i}^{\prime}\right]=\left[K_{j}, K_{j}\right] .
$$

Hence $H_{p} \cap K_{j} \neq\{1\}$, and so $j=q$ by the uniqueness of $q$. Therefore $K_{q} \subseteq W_{\ell}^{\prime} \cap W_{i}^{\prime}$, and so $i=\ell$ and $\ell$ is unique. 
By Lemma 3.5, we have $Z(W) W_{k}=Z(W) H_{p}=Z(W) K_{q}=Z(W) W_{\ell}^{\prime}$.

Suppose $\left|W_{k}\right|=\left|W_{\ell}^{\prime}\right|$. As $H_{p} \cong K_{q}$, we have either $W_{k}=H_{p}$ and $W_{\ell}^{\prime}=K_{q}$ or $W_{k}=H_{p-1} \times H_{p}$, with $\left|H_{p-1}\right|=2$, and $W_{\ell}^{\prime}=K_{q-1} \times K_{q}$, with $\left|K_{q-1}\right|=2$. Hence $W_{k} \cong W_{\ell}^{\prime}$ and $\left(W_{k}, S_{k}\right) \cong\left(W_{\ell}^{\prime}, S_{\ell}^{\prime}\right)$, since $\left(W_{k}, S_{k}\right)$ and $\left(W_{\ell}^{\prime}, S_{\ell}^{\prime}\right)$ are irreducible. Moreover $\pi_{q} \iota_{p}: H_{p} \rightarrow K_{q}$ is an isomorphism that restricts to the identity on $\left[H_{p}, H_{p}\right]$. If $W_{k}=H_{p}$ and $W_{\ell}^{\prime}=K_{q}$, let $\phi=\pi_{q} \iota_{p}$. If $W_{k}=H_{p-1} \times H_{p}$ and $W_{\ell}^{\prime}=K_{q-1} \times K_{q}$ extend $\pi_{q} \iota_{p}: H_{p} \rightarrow K_{q}$ to an isomorphism $\phi: W_{k} \rightarrow W_{\ell}^{\prime}$ by mapping the generator of $H_{p-1}$ to the generator of $H_{q-1}$. Then $\phi: W_{k} \rightarrow W_{\ell}^{\prime}$ is an isomorphism that restricts to the identity on $\left[W_{k}, W_{k}\right]=\left[H_{p}, H_{p}\right]$.

Suppose $\left|W_{k}\right|<\left|W_{\ell}^{\prime}\right|$. As $H_{p} \cong K_{q}$, we have $W_{k}=H_{p}$ and $W_{\ell}^{\prime}=K_{q-1} \times K_{q}$, with $\left|K_{q-1}\right|=2$. By Lemmas 3.1-3.4, either $\left(W_{k}, S_{k}\right) \cong \mathbf{B}_{2 q+1}$ and $\left(W_{\ell}^{\prime}, S_{\ell}^{\prime}\right) \cong \mathbf{C}_{2 q+1}$ for some $q \geq 1$ or $\left(W_{k}, S_{k}\right) \cong \mathbf{D}_{2}(2 q+1)$ and $\left(W_{\ell}^{\prime}, S_{\ell}^{\prime}\right) \cong \mathbf{D}_{2}(4 q+2)$ for some $q \geq 1$. Moreover $\pi_{q} \iota_{p}: H_{p} \rightarrow K_{q}$ is an isomorphism that restricts to the identity on $\left[H_{p}, H_{p}\right]$. Hence $\pi_{q} \iota_{p}: H_{p} \rightarrow K_{q}$ extends to a monomorphism $\phi: W_{k} \rightarrow W_{\ell}^{\prime}$ that restricts to the identity on $\left[W_{k}, W_{k}\right]$.

\section{The Basic Matching Theorem}

Let $(W, S)$ be a Coxeter system, and let $w$ be in $W$. The length of $w$ with respect to $S$, denoted by $\ell(w)$, is the least nonnegative integer $n$ such that $w$ can be written as a product $s_{1} s_{2} \cdots s_{n}$ of elements of $S$. A product $s_{1} s_{2} \cdots s_{n}$ of elements of $S$ is said to be reduced if $n=\ell\left(s_{1} s_{2} \cdots s_{n}\right)$. Every Coxeter system $(W, S)$ satisfies the deletion condition, which says that if a product $s_{1} s_{2} \cdots s_{n}$ of elements of $S$ is not reduced, then there are indices $i, j$, with $1 \leq i<j \leq n$ such that

$$
s_{1} s_{2} \cdots s_{n}=s_{1} \cdots s_{i-1} s_{i+1} \cdots s_{j-1} s_{j+1} \cdots s_{n} .
$$

The next lemma follows from the deletion condition.

Lemma 4.1 If $A \subseteq S$ and $w \in W$, then there is a unique shortest element $u$ of the coset $w\langle A\rangle$. Moreover an element $u$ of $w\langle A\rangle$ is the shortest element of $w\langle A\rangle$ if and only if $\ell(u a)>\ell(u)$ for each $a$ in $A$.

The next lemma follows from Lemma 4.1 and the deletion condition.

Lemma 4.2 (Bourbaki [1, Chapter IV.1 Exercise 3]) If $A, B \subseteq S$ and $w \in W$, then there is a unique shortest representative $u$ of the double coset $\langle A\rangle w\langle B\rangle$. Moreover an element $u$ of $\langle A\rangle w\langle B\rangle$ is the shortest element of $\langle A\rangle w\langle B\rangle$ if and only if $u$ is the shortest element of both $\langle A\rangle u$ and $u\langle B\rangle$. 
Lemma 4.3 Let $A, B \subseteq S$ and let $w \in W$ be such that $w\langle A\rangle w^{-1} \subseteq\langle B\rangle$. If $u$ is the shortest element of $\langle B\rangle w\langle A\rangle$, then $u A u^{-1} \subseteq B$ with equality if and only if $w\langle A\rangle w^{-1}=\langle B\rangle$.

Proof Certainly we have $u\langle A\rangle u^{-1} \subseteq\langle B\rangle$. Let $u=u_{1} \cdots u_{n}$ be reduced. For any $a$ in $A$, the word $u a=u_{1} \cdots u_{n} a$ is reduced by Lemma 4.1 and the deletion condition. Now $u a u^{-1}$ is in $\langle B\rangle$. Write $u a u^{-1}=b_{1} \cdots b_{k}$ with $b_{1} \cdots b_{k}$ reduced in $\langle B\rangle$. Now $u$ is a shortest element of $\langle B\rangle u$, and so $b_{1} \cdots b_{k} u_{1} \cdots u_{n}$ is reduced by the deletion condition. As $u_{1} \cdots u_{n} a=b_{1} \cdots b_{k} u_{1} \cdots u_{n}$, we have $k=1$ and $u a u^{-1}=b_{1}$. Thus $u A u^{-1} \subseteq B$. If $w\langle A\rangle w^{-1}=\langle B\rangle$, then $w^{-1}\langle B\rangle w=\langle A\rangle$ and $u^{-1}$ is the shortest element of $\langle A\rangle w^{-1}\langle B\rangle$. Therefore $u^{-1} B u \subseteq A$, and so $B \subseteq u A u^{-1}$. Hence $u A u^{-1}=B$.

Lemma 4.4 (Bourbaki [1, Chapter IV.1 Exercise 22]) Let $w_{0}$ be an element of $W$. Then the following are equivalent:

(1) $l\left(w_{0} s\right)<l\left(w_{0}\right)$ for all $s$ in $S$,

(2) $l\left(w_{0} w\right)=l\left(w_{0}\right)-l(w)$ for all $w$ in $W$.

Such an element $w_{0}$ is unique and exists if and only if $W$ is finite. If $W$ is finite, then $w_{0}$ is the unique element of maximal length in $W$. Moreover $w_{0}^{2}=1$ and $w_{0} S w_{0}=S$.

Let $(W, S)$ be a Coxeter system. The quasi-center of $(W, S)$ is the subgroup

$$
Q Z(W, S)=\left\{w \in W: w S w^{-1}=S\right\} .
$$

Lemma 4.5 (Bourbaki [1, Chapter V.4 Exercise 3]) Let $(W, S)$ be an irreducible Coxeter system with a nontrivial quasi-center. Then $W$ is a finite group and $Q Z(W)=$ $\left\{1, w_{0}\right\}$ with $w_{0}$ the longest element of $(W, S)$.

Let $V$ be a real vector space having a basis $\left\{e_{s}: s \in S\right\}$ in one-to-one correspondence with $S$. Let $B$ be the symmetric bilinear form on $V$ defined by

$$
B\left(e_{s}, e_{t}\right)= \begin{cases}-\cos (\pi / m(s, t)) & \text { if } m(s, t)<\infty \\ -1 & \text { if } m(s, t)=\infty\end{cases}
$$

There is an action of $W$ on $V$ defined by

$$
s(x)=x-2 B\left(x, e_{s}\right) e_{s} \text { for all } s \in S \text { and } x \in V .
$$

The root system of $(W, S)$ is the set of vectors

$$
\Phi=\left\{w\left(e_{s}\right): w \in W \text { and } s \in S\right\} .
$$

Algebraic 83 Geometric Topology, Volume 7 (2007) 
The elements of $\Phi$ are called roots. By Deodhar [7, Proposition 2.1], every root $\phi$ can be written uniquely in the form $\phi=\sum_{s \in S} a_{s} e_{s}$ with $a_{s} \in \mathbb{R}$ where either $a_{s} \geq 0$ for all $s$ or $a_{s} \leq 0$ for all $s$. In the former case, we say $\phi$ is positive and write $\phi>0$. By Deodhar [7, Proposition 2.2], if $w \in W$ and $s \in S$, then $w\left(e_{s}\right)>0$ if and only if $\ell(w s)>\ell(w)$. Let $\Phi^{+}$be the set of positive roots.

The set of reflections of $(W, S)$ is the set

$$
T=\left\{w s w^{-1}: w \in W \text { and } s \in S\right\} .
$$

By Deodhar [7, Proposition 3.1], the function $\rho: \Phi^{+} \rightarrow T$ defined by $\rho\left(w\left(e_{s}\right)\right)=$ $w s w^{-1}$ is well defined and a bijection.

If $A \subseteq S$, set $E_{A}=\left\{e_{s}: s \in A\right\}$. The next lemma follows from Lemma 4.1 and the above discussion of roots.

Lemma 4.6 If $A, B \subseteq S$ and $w \in W$, then $w\left(E_{A}\right)=E_{B}$ if and only if $w A w^{-1}=B$ and $w$ is the shortest element of $w\langle A\rangle$.

Suppose $A \subseteq S$. If $\langle A\rangle$ is finite, we denote the longest element of $\langle A\rangle$ by $w_{A}$. Suppose $s \in S-A$. Let $K \subseteq S$ be the component of $A \cup\{s\}$ containing $s$. We say that $s$ is $A$-admissible if $\langle K\rangle$ is finite. If $s$ is $A$-admissible, define

$$
v(s, A)=w_{K} w_{K-\{s\}} .
$$

Then $v(s, A)$ is the shortest element of $w_{K}\langle A\rangle$ by Lemma 4.4. Now $K-\{s\}$ is a union of components of $A$, and so $w_{K-\{s\}} A w_{K-\{s\}}=A$. Hence, if $t=w_{K} s w_{K}$ and $B=(A \cup\{s\})-\{t\}$, then $v(s, A)\left(E_{A}\right)=E_{B}$ by Lemma 4.6.

Proposition 4.7 (Deodhar [7, Proposition 5.5]) Let $A, B \subseteq S$, and let $w \in W$. If $w\left(E_{A}\right)=E_{B}$ and $w \neq 1$, then there exists a sequence $A_{1}, A_{2}, \ldots, A_{n+1}$ of subsets of $S$, and a sequence $s_{1}, s_{2}, \ldots, s_{n}$ of elements of $S$ such that

(1) $A_{1}=A$ and $A_{n+1}=B$,

(2) $s_{i} \in S-A_{i}$ and $s_{i}$ is $A_{i}$-admissible for $i=1, \ldots, n$,

(3) $v\left(s_{i}, A_{i}\right)\left(E_{A_{i}}\right)=E_{A_{i+1}}$ for $i=1, \ldots, n$,

(4) $w=v\left(s_{n}, A_{n}\right) \cdots v\left(s_{2}, A_{2}\right) v\left(s_{1}, A_{1}\right)$,

(5) $l(w)=l\left(v\left(s_{1}, A_{1}\right)\right)+l\left(v\left(s_{2}, A_{2}\right)\right)+\cdots+l\left(v\left(s_{n}, A_{n}\right)\right)$.

The next lemma follows from Proposition 4.7.

Algebraic 83 Geometric Topology, Volume 7 (2007) 
Lemma 4.8 Let $A \subseteq S$. Then there exists $B \subseteq S$ such that $A \neq B$ and $\langle A\rangle$ is conjugate to $\langle B\rangle$ in $W$ if and only if there exists $s \in S-A$ such that $m(s, a)>2$ for some $a \in A$, the element $s$ is $A$-admissible, and if $K$ is the component of $A \cup\{s\}$ containing $s$, then $w_{K} s w_{K} \neq s$.

Lemma 4.9 Let $A, B \subseteq S$ with $A$ irreducible and $\langle A\rangle$ conjugate to $\langle B\rangle$ in $W$. If $A$ is neither of type $\mathbf{A}_{n}$, for some $n$, nor of type $\mathbf{B}_{5}$, then $A=B$.

Proof Suppose $s \in S-A$, with $m(s, a)>2$ for some $a \in A$, and suppose $s$ is $A$-admissible. Then $K=A \cup\{s\}$ is irreducible and $\langle K\rangle$ is finite. By Lemma 4.8, it suffices to show that $w_{K} s w_{K}=s$. This is clear if $\left\langle w_{K}\right\rangle$ is the center of $\langle K\rangle$. Suppose that $Z(\langle K\rangle)=1$. Now $K$ is not of type $\mathbf{A}_{n+1}, \mathbf{D}_{2}(k)$, or $\mathbf{E}_{6}$, since $A$ is not of type $\mathbf{A}_{n}$ or $\mathbf{B}_{5}$. Hence $K$ must be of type $\mathbf{B}_{2 q+1}$ for some $q \geq 2$. Then $A$ is of type $\mathbf{B}_{2 q}$ and $w_{K} s w_{K}=s$.

Lemma 4.10 Let $A, B \subseteq S$. If $\langle A\rangle$ is a maximal (irreducible) finite visible subgroup of $(W, S)$ and $\langle A\rangle$ and $\langle B\rangle$ are conjugate, then $A=B$.

Proof Assume first that $\langle A\rangle$ is a maximal finite visible subgroup of $(W, S)$. If $s \in S-A$, then the component of $A \cup\{s\}$ containing $s$ is infinite. Hence no $s \in S-A$ is $A$-admissible, and so $A=B$ by Lemma 4.8.

Now assume that $\langle A\rangle$ is a maximal, irreducible, finite, visible subgroup of $(W, S)$. If $s \in S-A$ and $s$ is $A$-admissible, then $\{s\}$ is the component of $A \cup\{s\}$ containing $s$, and so $A=B$ by Lemma 4.8 .

Proposition 4.11 (Bourbaki [1, Chapter V.4, Exercise 2]) If $H$ is a finite subgroup of $W$, then there is a subset $A$ of $S$ such that $\langle A\rangle$ is finite and $H$ is conjugate to a subgroup of $\langle A\rangle$.

Lemma 4.12 Every maximal finite visible subgroup of $(W, S)$ is a maximal finite subgroup of $W$.

Proof Let $M \subseteq S$ be such that $\langle M\rangle$ is a maximal finite visible subgroup of $(W, S)$. Suppose $H$ is a finite subgroup of $W$ containing $\langle M\rangle$. Then $w H w^{-1} \subseteq\langle A\rangle$ for some $w \in W$ and some $A \subseteq S$ such that $\langle A\rangle$ is finite by Proposition 4.11. Then $w\langle M\rangle w^{-1} \subseteq\langle A\rangle$. Let $u$ be the shortest element of $\langle A\rangle w\langle M\rangle$. Then $u M u^{-1} \subseteq A$ by Lemma 4.3. Hence $u M u^{-1}=M$ by Lemma 4.10. Therefore $M=A$, since $M$ is a maximal finite visible subgroup. Hence $w\langle M\rangle w^{-1}=\langle A\rangle$ and so $\langle M\rangle=H$. Thus $\langle M\rangle$ is a maximal finite subgroup of $W$. 
A simplex $C$ of $(W, S)$ is a complete subset $C$ of $S$. A simplex $C$ of $(W, S)$ is said to be spherical if $\langle C\rangle$ is finite. The next proposition follows from Proposition 4.11, Lemma 4.10 and Lemma 4.12.

Proposition 4.13 Let $W$ be a finitely generated Coxeter group with two sets of Coxeter generators $S$ and $S^{\prime}$, and let $M$ be a maximal spherical simplex of $(W, S)$. Then there is a unique maximal spherical simplex $M^{\prime}$ of $\left(W, S^{\prime}\right)$ such that $\langle M\rangle$ and $\left\langle M^{\prime}\right\rangle$ are conjugate in $W$.

Proposition 4.14 (Solomon [13, Lemma 2]) Let $A, B \subseteq S$ and $w \in W$. Write $w=x u y$ with $x \in\langle A\rangle, y \in\langle B\rangle$, and $u$ the shortest element of $\langle A\rangle w\langle B\rangle$. Then

$$
\langle A\rangle \cap w\langle B\rangle w^{-1}=x\left\langle A \cap u B u^{-1}\right\rangle x^{-1} .
$$

Let $(W, S)$ be a Coxeter system of finite rank. A parabolic subgroup of $(W, S)$ is a subgroup of $W$ that is conjugate to a visible subgroup of $(W, S)$. By Proposition 4.14, any intersection of parabolic subgroups of $(W, S)$ is parabolic. The parabolic closure of a subset $X$ of $W$, denoted $\operatorname{Pc}(X)$, is the intersection of all the parabolic subgroups of $(W, S)$ that contain $X$. Then $\operatorname{Pc}(X)$ is the smallest parabolic subgroup of $(W, S)$ containing $X$. Clearly, if $w \in W$, then $\operatorname{Pc}\left(w X w^{-1}\right)=w \operatorname{Pc}(X) w^{-1}$.

Lemma 4.15 If $s, t$ in $S$ do not commute, then $\operatorname{Pc}(\{s t s t\})=\langle s, t\rangle$.

Proof Clearly, $\operatorname{Pc}(\{s t s t\}) \subseteq\langle s, t\rangle$. By Lemma 4.3, the rank of a parabolic subgroup of $(W, S)$ is well defined to be the rank of any visible subgroup conjugate to it. We have that $\operatorname{rank} \operatorname{Pc}(\{s t s t\})>0$, since $s t s t \neq 1$, and we have that $\operatorname{rank} \operatorname{Pc}(\{s t s t\})>1$, since stst is not a reflection. Therefore $\operatorname{Pc}(\{s t\})=\langle s, t\rangle$ by Lemma 4.3.

Lemma 4.16 If $A \subseteq S$ with no $a \in A$ central in $\langle A\rangle$, then

$$
\operatorname{Pc}([\langle A\rangle,\langle A\rangle])=\langle A\rangle .
$$

Proof Let $a \in A$. Then there exist $b \in A$ such that $a, b$ do not commute. By Lemma 4.15, we have

$$
\langle a, b\rangle=\operatorname{Pc}(\{a b a b\}) \subseteq \operatorname{Pc}([\langle A\rangle,\langle A\rangle]) \subseteq\langle A\rangle .
$$

Hence $A \subseteq \operatorname{Pc}([\langle A\rangle,\langle A\rangle])$, and so $\operatorname{Pc}([\langle A\rangle,\langle A\rangle])=\langle A\rangle$.

Lemma 4.17 Let $A, B \subseteq S$ with no $a \in A$ central in $\langle A\rangle$. Suppose

$$
w[\langle A\rangle,\langle A\rangle] w^{-1} \subseteq\langle B\rangle \text { for some } w \in W .
$$

Let $u$ be the shortest element of $\langle B\rangle w\langle A\rangle$. Then $u A u^{-1} \subseteq B$. 
Proof As $[\langle A\rangle,\langle A\rangle] \subseteq w^{-1}\langle B\rangle w$, we have that $\langle A\rangle \subseteq w^{-1}\langle B\rangle w$ by Lemma 4.16. Therefore $u A u^{-1} \subseteq B$ by Lemma 4.3.

Let $(W, S)$ be a Coxeter system. A basic subset of $S$ is a maximal irreducible subset $B$ of $S$ such that $\langle B\rangle$ is a noncyclic finite group. If $B$ is a basic subset of $S$, we call $B$ a base of $(W, S)$ and $\langle B\rangle$ a basic subgroup of $W$.

Theorem 4.18 (Basic Matching Theorem) Let $W$ be a finitely generated Coxeter group with two sets of Coxeter generators $S$ and $S^{\prime}$. Let $B$ be a base of $(W, S)$. Then there is a unique irreducible subset $B^{\prime}$ of $S^{\prime}$ such that $[\langle B\rangle,\langle B\rangle]$ is conjugate to $\left[\left\langle B^{\prime}\right\rangle,\left\langle B^{\prime}\right\rangle\right]$ in $W$. Moreover,

(1) the set $B^{\prime}$ is a base of $\left(W, S^{\prime}\right)$, and we say that $B$ and $B^{\prime}$ match,

(2) if $|\langle B\rangle|=\left|\left\langle B^{\prime}\right\rangle\right|$, then $B$ and $B^{\prime}$ have the same type and there is an isomorphism $\phi:\langle B\rangle \rightarrow\left\langle B^{\prime}\right\rangle$ that restricts to conjugation on $[\langle B\rangle,\langle B\rangle]$ by an element of $W$, and we say that $B$ and $B^{\prime}$ match isomorphically,

(3) if $|\langle B\rangle|<\left|\left\langle B^{\prime}\right\rangle\right|$, then either $B$ has type $\mathbf{B}_{2 q+1}$ and $B^{\prime}$ has type $\mathbf{C}_{2 q+1}$ for some $q \geq 1$ or $B$ has type $\mathbf{D}_{2}(2 q+1)$ and $B^{\prime}$ has type $\mathbf{D}_{2}(4 q+2)$ for some $q \geq 1$. Moreover, there is a monomorphism $\phi:\langle B\rangle \rightarrow\left\langle B^{\prime}\right\rangle$ that restricts to conjugation on $[\langle B\rangle,\langle B\rangle]$ by an element of $W$.

Proof Let $M \subseteq S$ be a maximal spherical simplex containing $B$. Then there is a unique maximal spherical simplex $M^{\prime}$ of $\left(W, S^{\prime}\right)$ and an element $u$ of $W$ such that $\left\langle M^{\prime}\right\rangle=u\langle M\rangle u^{-1}$ by Proposition 4.13. By the Matching Theorem for Systems of a Finite Coxeter Group applied to $\left(\left\langle M^{\prime}\right\rangle, u M u^{-1}\right)$ and $\left(\left\langle M^{\prime}\right\rangle, M^{\prime}\right)$, there is a base $B^{\prime}$ of $\left(\left\langle M^{\prime}\right\rangle, M^{\prime}\right)$ such that

$$
\left[\left\langle B^{\prime}\right\rangle,\left\langle B^{\prime}\right\rangle\right]=\left[\left\langle u B u^{-1}\right\rangle,\left\langle u B u^{-1}\right\rangle\right]=u[\langle B\rangle,\langle B\rangle] u^{-1} .
$$

Moreover, $B^{\prime}$ satisfies conditions 2 and 3, and so $|B|=\left|B^{\prime}\right|$.

Let $C^{\prime}$ be a base of $\left(W, S^{\prime}\right)$ that contains $B^{\prime}$. Then by the above argument, there is a $C \subseteq S$ and a $v \in W$ such that $\langle C\rangle$ is a finite irreducible subgroup of $(W, S)$, and $|C|=\left|C^{\prime}\right|$, and $[\langle C\rangle,\langle C\rangle]=v\left[\left\langle C^{\prime}\right\rangle,\left\langle C^{\prime}\right\rangle\right] v^{-1}$. Then we have $v u[\langle B\rangle,\langle B\rangle] u^{-1} v^{-1} \subseteq$ $[\langle C\rangle,\langle C\rangle]$. By Lemma 4.17, there is a $w \in W$ such that $w B w^{-1} \subseteq C$. As $B$ is a base of $(W, S)$, we have that $w B w^{-1}=C=B$ by Lemma 4.10. Therefore $B^{\prime}=C^{\prime}$ and $B^{\prime}$ is a base of $\left(W, S^{\prime}\right)$.

Suppose $D^{\prime} \subseteq S^{\prime}$ is irreducible and $x \in W$ such that $\left[\left\langle D^{\prime}\right\rangle,\left\langle D^{\prime}\right\rangle\right]=x[\langle B\rangle,\langle B\rangle] x^{-1}$. Then $x u^{-1}\left[\left\langle B^{\prime}\right\rangle,\left\langle B^{\prime}\right\rangle\right] u x^{-1}=\left[\left\langle D^{\prime}\right\rangle,\left\langle D^{\prime}\right\rangle\right]$. By Lemma 4.17, there is a $y \in W$ such that $y B^{\prime} y^{-1} \subseteq D^{\prime}$. As $B^{\prime}$ is a base of $\left(W, S^{\prime}\right)$ and $D^{\prime}$ is irreducible, $y B^{\prime} y^{-1}=D^{\prime}=B^{\prime}$ by Lemma 4.10 . Thus $B^{\prime}$ is unique. 
A group $G$ has property FA if for every tree on which $G$ acts without inversions, the set of fixed points of $G$ in the tree is nonempty. Let $(W, S)$ be a Coxeter system. If $A$ is a complete subset of $S$, we say that $\langle A\rangle$ is a complete visible subgroup of $(W, S)$.

Proposition 4.19 (Mihalik and Tschantz [10]) Let $(W, S)$ be a Coxeter system of finite rank. The maximal FA subgroups of $W$ are the conjugates of the maximal complete visible subgroups of $(W, S)$.

Lemma 4.20 Let $A, B \subseteq S$. If $\langle A\rangle$ is a maximal complete visible subgroup of ( $W, S)$ and $\langle A\rangle$ and $\langle B\rangle$ are conjugate, then $A=B$.

Proof If $s \in S-A$, then the component $K$ of $A \cup\{s\}$ containing $s$ is incomplete, and so $\langle K\rangle$ is infinite and $s$ is not $A$-admissible. Hence $A=B$ by Lemma 4.8.

The next proposition follows from Proposition 4.19 and Lemma 4.20.

Proposition 4.21 Let $W$ be a finitely generated Coxeter group with two sets of Coxeter generators $S$ and $S^{\prime}$, and let $M$ be a maximal simplex of $(W, S)$. Then there is a unique maximal simplex $M^{\prime}$ of $\left(W, S^{\prime}\right)$ such that $\langle M\rangle$ and $\left\langle M^{\prime}\right\rangle$ are conjugate in $W$.

\section{Visual graph of groups decompositions}

Let $(W, S)$ be a Coxeter system of finite rank. Suppose that $S_{1}, S_{2} \subseteq S$, with $S=S_{1} \cup S_{2}$ and $S_{0}=S_{1} \cap S_{2}$, are such that $m(a, b)=\infty$ for all $a \in S_{1}-S_{0}$ and $b \in S_{2}-S_{0}$. Then we can write $W$ as a visual amalgamated product

$$
W=\left\langle S_{1}\right\rangle *\left\langle S_{0}\right\rangle\left\langle S_{2}\right\rangle .
$$

We say that $S_{0}$ separates $S$ if $S_{1}-S_{0} \neq \varnothing$ and $S_{2}-S_{0} \neq \varnothing$. The amalgamated product decomposition of $W$ will be nontrivial if and only if $S_{0}$ separates $S$. If $S_{0}$ separates $S$, we call the triple $\left(S_{1}, S_{0}, S_{2}\right)$ a separation of $S$. Note that $S_{0}$ separates $S$ if and only if $S_{0}$ separates $\Gamma(W, S)$, that is, there are $a, b$ in $S-S_{0}$ such that every path in $\Gamma(W, S)$ from $a$ to $b$ must pass through $S_{0}$.

Let $\ell \in\left\langle S_{0}\right\rangle$ such that $\ell S_{0} \ell^{-1}=S_{0}$. By Lemma 4.5, we have $S_{0}=S_{\bullet} \cup\left(S_{0}-S_{\bullet}\right)$ where $S_{\bullet}$ generates a finite group, each element of $S_{\bullet}$ commutes with each element of $S_{0}-S_{\bullet}$, and $\ell$ is the longest element of $\left\langle S_{\bullet}\right\rangle$. The triple $\left(S_{1}, \ell, S_{2}\right)$ determines an elementary twist of $(W, S)$ (or of its P-diagram) giving a new Coxeter generating set 
$S_{*}=S_{1} \cup \ell S_{2} \ell^{-1}$ of $W$. Elementary twists of Coxeter systems were introduced by Brady et al [2].

In application, it is simpler to consider a more general kind of twisting. Suppose $S_{0}$ and $\bar{S}_{0} \subseteq S_{2}$ generate conjugate subgroups of $\left\langle S_{2}\right\rangle$. Suppose $d \in\left\langle S_{2}\right\rangle$ is such that $d \bar{S}_{0} d^{-1}=S_{0}$. Then $S_{1} \cap d S_{2} d^{-1}=S_{0}$, since

$$
S_{0} \subseteq S_{1} \cap d S_{2} d^{-1} \subseteq S_{1} \cap\left\langle S_{2}\right\rangle=S_{0} .
$$

A generalized twist (or simply twist) of $(W, S)$ in this situation gives a new Coxeter generating set $S_{*}=S_{1} \cup d S_{2} d^{-1}$ of $W$ and a new visual amalgamated product decomposition $W=\left\langle S_{1}\right\rangle *\left\langle S_{0}\right\rangle\left\langle d S_{2} d^{-1}\right\rangle$.

Elementary and generalized twists can be easily understood in terms of their effects on $\mathrm{P}$-diagrams. The $\mathrm{P}$-diagram of $(W, S)$ is the union of the $\mathrm{P}$-diagrams for $\left\langle S_{1}\right\rangle$ and $\left\langle S_{2}\right\rangle$ overlapping in the $\mathrm{P}$-diagram for $\left\langle S_{0}\right\rangle$. The $\mathrm{P}$-diagram for $\left(W, S_{*}\right)$ is obtained from the $\mathrm{P}$-diagram of $(W, S)$ by twisting the $\mathrm{P}$-diagram of $\left\langle S_{2}\right\rangle$, that is, removing the $\mathrm{P}$-diagram for $\left\langle S_{2}\right\rangle$, replacing it by the isomorphic $\mathrm{P}$-diagram of $\left\langle d S_{2} d^{-1}\right\rangle$, and attaching it to the P-diagram for $\left\langle S_{1}\right\rangle$ along $S_{0}=d \bar{S}_{0} d^{-1}$. If $S_{0}=S_{1}$ or $S_{0}=S_{2}$, we call the twist degenerate. A degenerate twist does not change the isomorphism type of the P-diagram. This includes the case where $S_{0}=S_{1}=\varnothing, S_{2}=S$, giving $S_{*}=d S d^{-1}$ the conjugation of $S$ by an arbitrary $d \in W$.

Proposition 5.1 (Brink and Howlett [3, Proposition 2.1]) If $(W, S)$ is a Coxeter system and $A \subseteq S$, then the normalizer of $\langle A\rangle$ in $W$ is the semidirect product of $\langle A\rangle$ by $\left\{w \in W: w\left(E_{A}\right)=E_{A}\right\}$.

Proposition 5.2 Any generalized twist of a Coxeter system $(W, S)$ can be realized by a sequence of elementary twists.

Proof Suppose $S_{1}, S_{2} \subseteq S$, with $S=S_{1} \cup S_{2}$ and $S_{0}=S_{1} \cap S_{2}$, and $m(a, b)=\infty$ for all $a \in S_{1}-S_{0}$ and $b \in S_{2}-S_{0}$. Suppose $\bar{S}_{0} \subseteq S_{2}$ and there is an $d \in\left\langle S_{2}\right\rangle$ such that $d \bar{S}_{0} d^{-1}=S_{0}$. Let $\bar{d}$ be the shortest element of $\left\langle S_{0}\right\rangle d\left\langle\bar{S}_{0}\right\rangle$. Then $\bar{d} \bar{S}_{0} \bar{d}^{-1}=S_{0}$ by Lemma 4.3 and $\bar{d}\left(E_{\bar{S}_{0}}\right)=E_{S_{0}}$ by Lemma 4.6. Hence $d \bar{d}^{-1} S_{0} \bar{d} d^{-1}=S_{0}$. By Proposition 5.1, there is a $u \in\left\langle S_{0}\right\rangle$ and a $v \in\left\langle S_{2}\right\rangle$ such that $d \bar{d}^{-1}=u v$ and $v\left(E_{S_{0}}\right)=E_{S_{0}}$. Then $v S_{0} v^{-1}=S_{0}$ by Lemma 4.6. Now $u=d \bar{d}^{-1} v^{-1}$, and so $u S_{0} u^{-1}=S_{0}$. We have $\bar{d}^{-1} v^{-1}\left(E_{S_{0}}\right)=E_{\bar{S}_{0}}$. If $\bar{d}^{-1} v^{-1}=1$, then $d=u$ and $\left(S_{1}, d, S_{2}\right)$ determines an elementary twist. Suppose $\bar{d}^{-1} v^{-1} \neq 1$. By Proposition 4.7, there is a sequence $A_{1}, \ldots, A_{n+1}$ of subsets of $S_{2}$ and a sequence $s_{1}, \ldots, s_{n}$ of elements of $S_{2}$ such that $A_{1}=S_{0}, A_{n+1}=\bar{S}_{0}, s_{i} \in S_{2}-A_{i}$ and $s_{i}$ is $A_{i}-$ admissible for each $i$, and if $v_{i}=v\left(s_{i}, A_{i}\right)$ for each $i$, then $v_{i} A_{i} v_{i}^{-1}=A_{i+1}$ for each $i=1, \ldots, n$, and $\bar{d}^{-1} v^{-1}=v_{n} \cdots v_{1}$. Then $d=u v \bar{d}=u v_{1}^{-1} \cdots v_{n}^{-1}$. 
Let $K_{i}$ be the component of $A_{i} \cup\left\{s_{i}\right\}$ containing $s_{i}$. Then $v_{i}=w_{K_{i}} w_{K_{i}-\left\{s_{i}\right\}}$ and $w_{K_{i}-\left\{s_{i}\right\}} A_{i} w_{K_{i}-\left\{s_{i}\right\}}=A_{i}$ for each $i$. Now

$$
\left(S_{1} \cup\left\{s_{1}\right\}\right) \cap S_{2}=S_{0} \cup\left\{s_{1}\right\} \text { and } w_{K_{1}}\left(S_{0} \cup\left\{s_{1}\right\}\right) w_{K_{1}}=S_{0} \cup\left\{s_{1}\right\} .
$$

Therefore the triple $\left(S_{1} \cup\left\{s_{1}\right\}, w_{K_{1}}, S_{2}\right)$ determines an elementary twist of $(W, S)$ that yields the Coxeter generating set $S_{1} \cup w_{K_{1}} S_{2} w_{K_{1}}$. Now

$$
\left(S_{1} \cup\left\{s_{1}\right\}\right) \cap w_{K_{1}} S_{2} w_{K_{1}}=S_{0} \cup\left\{s_{1}\right\},
$$

and so $S_{1} \cap w_{K_{1}} S_{2} w_{K_{1}}=S_{0}$, since $s_{1} \in S_{2}-S_{0}$. Now $w_{K_{1}-\left\{s_{1}\right\}} S_{0} w_{K_{1}-\left\{s_{1}\right\}}=$ $S_{0}$, and so the triple $\left(S_{1}, w_{K_{1}-\left\{s_{1}\right\}}, w_{K_{1}} S_{2} w_{K_{1}}\right)$ determines an elementary twist of $\left(W, S_{1} \cup w_{K_{1}} S_{2} w_{K_{1}}\right)$ that yields the Coxeter generating set $S_{1} \cup v_{1}^{-1} S_{2} v_{1}$ with $S_{1} \cap v_{1}^{-1} S_{2} v_{1}=S_{0}$.

Let $s_{2}^{\prime}=v_{1}^{-1} s_{2} v_{1}$. Then $\left(S_{1} \cup\left\{s_{2}^{\prime}\right\}\right) \cap\left(v_{1}^{-1} S_{2} v_{1}\right)=S_{0} \cup\left\{s_{2}^{\prime}\right\}$. Observe that $v_{1}^{-1} w_{K_{2}} v_{1} \in\left\langle S_{0} \cup\left\{s_{2}^{\prime}\right\}\right\rangle$ and

$$
v_{1}^{-1} w_{K_{2}} v_{1}\left(S_{0} \cup\left\{s_{2}^{\prime}\right\}\right) v_{1}^{-1} w_{K_{2}} v_{1}=S_{0} \cup\left\{s_{2}^{\prime}\right\},
$$

and so the triple $\left(S_{1} \cup\left\{s_{2}^{\prime}\right\}, v_{1}^{-1} w_{K_{2}} v_{1}, v_{1}^{-1} S_{2} v_{1}\right)$ determines an elementary twist of $\left(W, S_{1} \cup v_{1}^{-1} S_{2} v_{1}\right)$ that yields the Coxeter generating set

$$
S_{1} \cup\left(v_{1}^{-1} w_{K_{2}} S_{2} w_{K_{2}} v_{1}\right) \text { with }\left(S_{1} \cup\left\{s_{2}^{\prime}\right\}\right) \cap\left(v_{1}^{-1} w_{K_{2}} S_{2} w_{K_{2}} v_{1}\right)=S_{0} \cup\left\{s_{2}^{\prime}\right\} .
$$

Now $S_{1} \cap\left(v_{1}^{-1} w_{K_{2}} S_{2} w_{K_{2}} v_{1}\right)=S_{0}$, since $s_{2}^{\prime} \in v_{1}^{-1} S_{2} v_{1}-S_{0}$.

Next observe that $v_{1}^{-1} w_{K_{2}-\left\{s_{2}\right\}} v_{1} \in\left\langle S_{0}\right\rangle$ and

$$
v_{1}^{-1} w_{K_{2}-\left\{s_{2}\right\}} v_{1} S_{0} v_{1}^{-1} w_{K_{2}-\left\{s_{2}\right\}} v_{1}=S_{0}
$$

Hence the triple

$$
\left(S_{1}, v_{1}^{-1} w_{K_{2}-\left\{s_{2}\right\}} v_{1}, v_{1}^{-1} w_{K_{2}} S_{2} w_{K_{2}} v_{1}\right)
$$

determines an elementary twist of $\left(W, S_{1} \cup v_{1}^{-1} w_{K_{2}} S_{2} w_{K_{2}} v_{1}\right)$ that yields the Coxeter generating set

$$
S_{1} \cup\left(v_{1}^{-1} v_{2}^{-1} S_{2} v_{1} v_{2}\right) \text { with } S_{1} \cap\left(v_{1}^{-1} v_{2}^{-1} S_{2} v_{1} v_{2}\right)=S_{0} .
$$

Continuing in this way, we will arrive at the Coxeter generating set

$$
S_{1} \cup\left(v_{1}^{-1} \cdots v_{n}^{-1} S_{2} v_{n} \cdots v_{1}\right) \text { with } S_{1} \cap\left(v_{1}^{-1} \cdots v_{n}^{-1} S_{2} v_{n} \cdots v_{1}\right)=S_{0} .
$$

Finally, the triple

$$
\left(S_{1}, u, v_{1}^{-1} \cdots v_{n}^{-1} S_{2} v_{n} \cdots v_{1}\right)
$$


determines an elementary twist of $\left(W, S_{1} \cup v_{1}^{-1} \cdots v_{n}^{-1} S_{2} v_{n} \cdots v_{1}\right)$ that yields the Coxeter generating set $S_{1} \cup d S_{2} d^{-1}$. Thus the generalized twist of $(W, S)$ determined by $\left(S_{1}, d, S_{2}\right)$ is a composition of elementary twists.

Let $\Lambda$ be a visual graph of groups decomposition of $(W, S)$. Then the graph of $\Lambda$ is a tree, since the abelianization of $W$ is finite. The graph of groups decomposition $\Lambda$ can be understood as a visual amalgamated product in many ways, eg by taking some of the vertex and edge groups to be generated by $S_{1}$, others to be generated by $S_{2}$, with the overlap being a single edge group of $\Lambda$. Hence we will also speak of twisting a visual graph of groups decomposition with respect to some such partitioning of the graph of groups and some conjugating element.

A graph of groups decomposition is said to be reduced if no edge group is equal to an incident vertex group. Suppose $\Lambda$ is a reduced visual graph of groups decomposition of a Coxeter system $(W, S)$ of finite rank. Suppose (for simplicity in this application) that no edge group of $\Lambda$ is a proper subgroup of another edge group of $\Lambda$. Construct another visual graph of groups decomposition (though not reduced) $\widetilde{\Lambda}$ as follows. The vertices of $\tilde{\Lambda}$ are of two distinct types, v-vertices and e-vertices. The v-vertices correspond to the vertices of $\Lambda$, and the e-vertices correspond to the distinct edge groups of $\Lambda$. An edge of $\tilde{\Lambda}$ will connect vertices $p$ and $q$ if $p$ is a v-vertex and $q$ is an e-vertex, and $p$ corresponds to an endpoint of an edge of $\Lambda$ with edge group corresponding to $q$. The vertex group of a v-vertex $p$ of $\tilde{\Lambda}$ will be the vertex group for $p$ in $\Lambda$. The vertex group of an e-vertex $q$ of $\tilde{\Lambda}$ is the edge group of $\Lambda$ corresponding to $q$. Each edge of $\widetilde{\Lambda}$, say from $p$ to $q$, will have edge group equal to the edge group of $\Lambda$ corresponding to the e-vertex $q$ of that edge. The maps of edge groups into vertex groups in $\tilde{\Lambda}$ will be inclusion maps (as in visual decompositions generally, determined by which of the Coxeter generators lie in each vertex and edge groups). Then by a series of reductions and expansions (inverse reductions) we can get from $\tilde{\Lambda}$ to $\Lambda$ and we see that they are both visual graph of group decompositions of $W$ (or by comparing the relations defining the fundamental groups of $\Lambda$ and $\tilde{\Lambda}$ ). On the other hand, different reduced visual graphs of groups $\Lambda$ and $\Lambda_{2}$ will correspond to the same $\tilde{\Lambda}=\tilde{\Lambda}_{2}$ provided they have the same vertex groups and edge groups, since the inclusion of edge groups into vertex groups determine the edges of $\widetilde{\Lambda}$ when no edge group is a proper subgroup of another edge group. The point here is that $\tilde{\Lambda}$ provides a way of keeping track of which edge groups of $\Lambda$ are equal and in which vertex groups without specifying what the subtree of edges of $\Lambda$ with the same given edge group must look like, (in essence, without specifying the order of the vertex groups containing this edge group). We think of $\tilde{\Lambda}$ as a flattened form of $\Lambda$ making uniform the relationship between vertex groups and different edge groups of $\Lambda$.

Algebraic $8 \mathcal{G}$ Geometric Topology, Volume 7 (2007) 
A particularly simple case is when the edge groups of $\Lambda$ are all equal. Then $\tilde{\Lambda}$ has one v-vertex for each vertex $p$ of $\Lambda$ and one e-vertex $q$ for the common edge group $E$ with edges from $q$ to $p$ for each v-vertex $p$ and with edge groups equal to $E$ and inclusion maps into the vertex groups. The fundamental group of $\Lambda$ is an amalgamated product of all the vertex groups of $\Lambda$ identifying the copies of the edge group in each vertex group.

\section{The Decomposition Matching Theorem}

If $U$ is a subgroup of $W$, write $U^{*}=\left\{w U w^{-1}: w \in W\right\}$ for the set of all subgroups conjugate to $U$ in $W$. Write $U^{*} \preceq V^{*}$ if for some $w \in W, U \subseteq w V w^{-1}$ (independent of the representatives for the conjugacy classes). Clearly $\preceq$ is transitive and reflexive. Consider the conjugacy classes of a visible subgroup $U$ and any subgroup $V$ of a Coxeter system $(W, S)$. If $U^{*} \preceq V^{*}$ and $V^{*} \preceq U^{*}$ then $U^{*}=V^{*}$, since if $U \subseteq w V w^{-1} \subseteq w z U z^{-1} w^{-1}$ then, since $U$ is a visible subgroup, $w z U z^{-1} w^{-1}=U$ and $U$ and $V$ are conjugate by Lemma 4.3. Hence for the conjugacy classes of visible subgroups, $\preceq$ is a partial order. We say that $J \subseteq S$ is a c-minimal separating subset of $S$ if $J$ separates $S$ and $\langle J\rangle^{*}$ is a $\preceq$-minimal element of the set of conjugacy classes of subgroups generated by separating subsets of $S$. Assuming there are separating subsets of $S$, there are finitely many since $S$ is finite, and so there are c-minimal separating subsets of $S$.

Theorem 6.1 Let $W$ be a finitely generated Coxeter group with two sets of Coxeter generators $S$ and $S^{\prime}$. If $(W, S)$ is complete, then $\left(W, S^{\prime}\right)$ is complete; otherwise, for any given nontrivial splitting $A *_{C} B$ of $W$, there exist $S_{0} \subseteq S, S_{0}^{\prime} \subseteq S^{\prime}$, a reduced visual graph of groups decomposition $\Lambda$ for $(W, S)$, and a reduced visual graph of groups decomposition $\Lambda^{\prime}$ for $\left(W, S^{\prime}\right)$ such that

(1) the set $S_{0}$ is a c-minimal separating subset of $S$, and $S_{0}^{\prime}$ is a c-minimal separating subset of $S^{\prime}$, with $\left\langle S_{0}\right\rangle^{*}=\left\langle S_{0}^{\prime}\right\rangle^{*} \preceq C^{*}$,

(2) the edge groups of $\Lambda$ are conjugate to $\left\langle S_{0}\right\rangle$, the edge groups of $\Lambda^{\prime}$ are conjugate to $\left\langle S_{0}^{\prime}\right\rangle$ (and hence are conjugate and conjugate to a subgroup of $C$ ) and

(3) there is a 1-1 correspondence between the vertices of $\Lambda$ and the vertices of $\Lambda^{\prime}$ such that each vertex group of $\Lambda$ is conjugate to the corresponding vertex group of $\Lambda^{\prime}$.

Proof If $(W, S)$ is complete, then $\left(W, S^{\prime}\right)$ is complete by Proposition 4.21. Suppose $(W, S)$ is incomplete. Given a nontrivial splitting $W=A *_{C} B$, there is some visual 
splitting $W=A_{1} * C_{1} B_{1}$, with respect to $S$, with $C_{1}$ a subgroup of a conjugate of $C$ by the visual decomposition theorem and [10, Remark 1]. Consider the finite collection of conjugacy classes $\langle J\rangle^{*}$, partially ordered by $\preceq$, for subsets $J \subseteq S$ such that $\langle J\rangle^{*} \preceq C^{*}$ and there is a visual splitting $W=A_{2} *\langle J\rangle B_{2}$. Then there exists such a $J$ with $\langle J\rangle^{*}$ minimal in this partial order.

Now starting with a splitting $W=A_{2} *\langle J\rangle B_{2}$ and working with respect to $S^{\prime}$, as above, there is a $J^{\prime} \subseteq S^{\prime}$ with $\left\langle J^{\prime}\right\rangle^{*} \preceq\langle J\rangle^{*}$ and a visual splitting $W=A_{3} *\left\langle J^{\prime}\right\rangle B_{3}$, with $\left\langle J^{\prime}\right\rangle^{*} \preceq$-minimal for such splitting $S^{\prime}$-visible subgroups.

Working back again from $W=A_{3} *\left\langle J^{\prime}\right\rangle B_{3}$ and splitting visually with respect to $S$, there is a $J^{\prime \prime} \subseteq S$ with $\left\langle J^{\prime \prime}\right\rangle^{*} \preceq\left\langle J^{\prime}\right\rangle^{*}$ and an $S$-visual splitting over $\left\langle J^{\prime \prime}\right\rangle$ with $\left\langle J^{\prime \prime}\right\rangle^{*} \preceq$-minimal. Now $\left\langle J^{\prime \prime}\right\rangle^{*} \preceq\left\langle J^{\prime}\right\rangle^{*} \preceq\langle J\rangle^{*} \preceq C^{*}$ but $J$ was taken so $\langle J\rangle^{*}$ was $\preceq$-minimal below $C^{*}$ having an $S$-visual splitting over $\langle J\rangle$, hence $\left\langle J^{\prime \prime}\right\rangle^{*}=\langle J\rangle^{*}$ (but not necessarily $\left\langle J^{\prime \prime}\right\rangle=\langle J\rangle$ ), and so in fact $\langle J\rangle^{*}=\left\langle J^{\prime}\right\rangle^{*}$ and (1) holds with $S_{0}=J$ and $S_{0}^{\prime}=J^{\prime}$.

Since $S$ is finite and each vertex group of a reduced visual graph of groups decomposition of $W$ is generated by a different subset of $S$, there is an obvious limit to the number of vertices in a reduced visual graph of groups decomposition of $W$, and in some sense, the more vertices, the finer the graph of groups decomposition. Take a reduced $S$-visual graph of groups decomposition $\Lambda$ of $W$ such that every edge group is conjugate to $\langle J\rangle$ and, among such, having a maximum number of vertices. By the visual decomposition theorem, take $\Lambda^{\prime}$ a reduced $S^{\prime}$-visual graph of groups decomposition refining $\Lambda$, ie such that each vertex (edge) group of $\Lambda^{\prime}$ is a subgroup of a conjugate of a vertex (edge) group of $\Lambda$. Similarly, take $\Lambda^{\prime \prime}$ a reduced $S$-visual graph of groups decomposition of $W$ refining $\Lambda^{\prime}$. The edge groups of $\Lambda^{\prime \prime}$ are equal to conjugates of the edge groups of $\Lambda$ by the c-minimality of $S_{0}$, and so are conjugate to the edge groups of $\Lambda^{\prime}$, and so (2) holds. We postpone the proof of (3) until after the proof of Lemma 6.3.

The following lemma characterizes the visual decomposition $\Lambda$.

Lemma 6.2 Let $(W, S)$ be a Coxeter system of finite rank, and let $J$ be a c-minimal separating subset of $S$. Let $\mathcal{E}$ be the set of separating subsets of $S$ that are conjugate to $J$ in $W$, and let $\mathcal{V}$ be the set of all maximal subsets of $S$ that are not separated by a set in $\mathcal{E}$. Let $\Lambda$ be a reduced visual graph of groups decomposition of $(W, S)$ having edge groups generated by conjugates of $J$ (and hence elements of $\mathcal{E}$ ) and among such has a maximum number of vertices. Then all of the subgroups generated by sets in $\mathcal{V}$ are the vertex groups of $\Lambda$, and all of the subgroups generated by sets in $\mathcal{E}$ are the edge groups of $\Lambda$. 
Proof By Lemma 4.9, all the visible conjugates of $\langle J\rangle$ are visual direct products $F \times G$ with conjugate finite factors $F$ and the same factor $G$ in common with all the visible conjugates of $\langle J\rangle$. If we split $W$ by a separating visible conjugate of $J$, each of the other visible conjugates of $J$ lies entirely in one of the factors of the free product with amalgamation, since the corresponding finite group $F$ lies in one factor and the group $G$ lies in each factor.

Assume $\Lambda$ is a reduced visual graph of groups with edge groups conjugate to $\langle J\rangle$ and among such having a maximal number of vertices. Note that each edge group, and hence each vertex group, contains the common subgroup $G$. The graph of $\Lambda$ is a tree, since the abelianization of $W$ is finite.

Suppose $L$ is the set of generators of a vertex group $V$ of $\Lambda$. We claim that $L$ is not separated by a set in $\mathcal{E}$. On the contrary, suppose $L$ is separated by a set $K$ in $\mathcal{E}$, say $x$ and $y$ are in different components of the P-diagram of $\langle L-K\rangle$. We claim that $K \subseteq L$. On the contrary, suppose $K \nsubseteq L$. Then $L \cap K$ does not separate $S$, by c-minimality of $J$, and so there is a path in the P-diagram of $(W, S)$ from $x$ to $y$ that avoids $L \cap K$. Take a path from $x$ to $y$ which is in a union of as few vertex groups of $\Lambda$ as possible. Let $V^{\prime}$ be a vertex group of $\Lambda$ containing a generator in this path not in $V$. Then the path passes through some edge group $E$ of $V$ at some first point before $V^{\prime}$ and must pass back through $E$ at some last point, since the graph of $\Lambda$ is a tree. Neither of these points is a generator of $G$ since these all lie in $L \cap K$. Hence these points are generators in the finite factor $F$ of $E$. But the P-diagram of $F$ is complete, and so there is a short circuit of the path going from the first to the last point in $F$ avoiding $V^{\prime}$. We conclude instead that the path hitting the fewest vertex groups of $\Lambda$ is a path in $L-K$, contradicting the assumption that $K$ separates $L$. Hence $K \subseteq L$ and there is a separation $\left(L_{1}, K, L_{2}\right)$ of $L$. Each edge group of $\Lambda$ incident to the vertex group $V=\langle L\rangle$ is contained in either the subgroup generated by $L_{1}$ or by $L_{2}$, and so we can split $V$ into two vertices generated by $L_{1}$ and $L_{2}$, respectively, and joined by an edge group generated by $K$, with each component of the rest of $\Lambda$ attached to one or the other of the new vertex groups by an edge group of $\Lambda$. Neither of the new vertex groups equals an incident edge group $E$, since the finite Coxeter groups $E / G$ and $\langle K\rangle / G$ have the same rank. This gives a reduced visual graph of groups decomposition over separating conjugates of $J$ with more vertex groups, contradicting the maximality of the number of vertices in $\Lambda$. Hence $L$ cannot be separated by a set in $\mathcal{E}$ as claimed. Clearly, every subset of $S$ that contains $L$ properly is separated by the set of generators of some edge group of $\Lambda$ that is incident to $V$. Therefore $L$ is a maximal subset of $S$ that is not separated by a set in $\mathcal{E}$, and so $L \in \mathcal{V}$.

Now suppose $L \in \mathcal{V}$. We claim that $\langle L\rangle$ is a vertex group of $\Lambda$. Every element of $L$ is a generator of some vertex group of $\Lambda$. Suppose $L^{\prime} \subseteq L$ is a maximal subset 
of $L$ contained in some vertex group of $\Lambda$. If $L-L^{\prime} \neq \varnothing$, say $x \in L-L^{\prime}$, then $L^{\prime}$ and $x$ are not both contained in a vertex group of $\Lambda$. Take vertex groups $V$ and $V^{\prime}$ of $\Lambda$, with $x \in V$ and $L^{\prime} \subseteq V^{\prime}$, which are closest together in the graph of $\Lambda$. Let $E$ be an edge group of the path between $V$ and $V^{\prime}$. Then $E$ is generated by a visible conjugate $K$ of $J$ which separates the generators in $V-E$ from those in $V^{\prime}-E$, and so $K \in \mathcal{E}$. Now $x \notin E$ otherwise $x$ would also be in a vertex group closer to $V^{\prime}$ on the path between $V$ and $V^{\prime}$. Likewise, $L^{\prime} \nsubseteq E$ or else $L^{\prime}$ would be contained in a vertex group closer to $V$ on a path between $V$ and $V^{\prime}$. But then the P-diagram of $\langle L-K\rangle$ would have at least two components, one containing $x$ and one containing some element of $L^{\prime}-K$. This contradicts the assumption that $L \in \mathcal{V}$. Instead all of $L$ must be contained in a vertex group $V$ of $\Lambda$. As the set of generators in $V$ is in $\mathcal{V}$, we have that $\langle L\rangle=V$.

Finally, suppose $K \in \mathcal{E}$. Then there is a separation $\left(S_{1}, K, S_{2}\right)$ of $S$. Each $L \in \mathcal{V}$ generates a vertex group of $\Lambda$ but is not separated by $K$ by our previous argument, and so each $L \in \mathcal{V}$ is contained in either $S_{1}$ or $S_{2}$. Pick vertex groups $V_{1}$ and $V_{2}$ as close together in $\Lambda$ as possible such that $V_{1}$ is generated by a subset of $S_{1}$ and $V_{2}$ is generated by a subset of $S_{2}$. Then $V_{1}$, and $V_{2}$ are adjacent since every vertex group in a path between these is generated by a subset of either $S_{1}$ or $S_{2}$. Now $V_{1} \cap V_{2}$ is an edge group $E$ of $\Lambda$ which is generated by a subset of $K$ but not by a proper subset of $K$ by the c-minimality of $J$, and so $E=\langle K\rangle$.

The next lemma explains the relationship between the visual decompositions $\Lambda$ and $\Lambda^{\prime \prime}$ of $(W, S)$.

Lemma 6.3 Let $\Lambda$ and $\Lambda^{\prime \prime}$ be reduced visual graph of groups decompositions of a Coxeter system $(W, S)$ of finite rank. Suppose the edge groups of $\Lambda$ are generated by conjugates of a c-minimal separating subset $J$ of $S$, and among visual decompositions with this same conjugacy class of edge groups, $\Lambda$ has a maximum number of vertex groups. Suppose each vertex and edge group of $\Lambda^{\prime \prime}$ is a subgroup of a conjugate of a vertex or edge group of $\Lambda$, respectively. Then the vertex and edge groups of $\Lambda$ are equal to the vertex and edge groups of $\Lambda^{\prime \prime}$, respectively, that is, $\tilde{\Lambda}=\widetilde{\Lambda}^{\prime \prime}$.

Proof By the last lemma, the vertex groups of $\Lambda$ are determined from the set of all separating sets of $S$ that are conjugate to $J$. Each edge group of $\Lambda^{\prime \prime}$ is generated by a separating subset of $S$ and is contained in a conjugate of $\langle J\rangle$, and so, by the c-minimality of $J$, must be a conjugate of $\langle J\rangle$ and an edge group also of $\Lambda$.

Let $T$ be the Bass-Serre tree with standard transversal $T_{*}$, corresponding to the graph of groups $\Lambda$, ie, the vertices of $T$ are the cosets of each vertex group of $\Lambda$ and $T_{*}$ 
consists of the cosets of each vertex group that contain the identity. A vertex group $G$ of $\Lambda^{\prime \prime}$ stabilizes a vertex $V$ of $T$, since $G$ is a subgroup of a conjugate of a vertex group of $\Lambda$. But each generator of $G$ also stabilizes a vertex of $T_{*}$ and the geodesic path from that vertex of $T_{*}$ to $V$. Hence $G$ also stabilizes the vertex of $T_{*}$ nearest to $V$. Thus each vertex group of $\Lambda^{\prime \prime}$ is actually a subgroup of a vertex group of $\Lambda$.

As the vertex groups of $\Lambda$ are proper subgroups, $\Lambda^{\prime \prime}$ has at least two vertices, and each vertex group of $\Lambda^{\prime \prime}$ contains an edge group of $\Lambda^{\prime \prime}$, which is a conjugate of $\langle J\rangle$, as a proper subgroup. Hence no vertex group of $\Lambda^{\prime \prime}$ is contained in an edge group of $\Lambda$, since all the visible conjugates of $\langle J\rangle$ have the same rank. Consequently, each vertex group of $\Lambda^{\prime \prime}$ can be contained in only one vertex group of $\Lambda$, otherwise a vertex group of $\Lambda^{\prime \prime}$ would be contained in the intersection of vertex groups for two different vertices of $\Lambda$ and so would be contained in each edge group for edges of $\Lambda$ in the geodesic path between these vertices, which is not the case.

Summarizing, for each vertex $U$ of $\Lambda^{\prime \prime}$, there exists a unique vertex $f(U)$ of $\Lambda$ such that the vertex group $\Lambda^{\prime \prime}(U)$ of $\Lambda^{\prime \prime}$ at $U$ is a subgroup of the vertex group $\Lambda(f(U))$ of $\Lambda$ at $f(U)$. We claim that for each vertex $V$ of $\Lambda$, the vertex group $\Lambda(V)$ is generated by the vertex groups of $\Lambda^{\prime \prime}$ for vertices in $f^{-1}(V)$. In particular, there will be at least one vertex of $\Lambda^{\prime \prime}$ in $f^{-1}(V)$, and so at least as many vertices in $\Lambda^{\prime \prime}$ as in $\Lambda$. But $\Lambda$ has a maximal number of vertices for visual reduced graph of groups decompositions of $(W, S)$ with edge groups that are conjugates of $\langle J\rangle$, so $\Lambda^{\prime \prime}$, which also satisfies these conditions, has no more vertices than $\Lambda$. Hence $\Lambda$ and $\Lambda^{\prime \prime}$ have the same number of vertices; moreover, for each vertex $V$ of $\Lambda$, we conclude that $f^{-1}(V)$ is a unique vertex of $\Lambda^{\prime \prime}$, and the vertex groups of these vertices in $\Lambda$ and $\Lambda^{\prime \prime}$ must be equal. Hence the vertex and edge groups of $\Lambda$ are the same as the vertex and edge groups of $\Lambda^{\prime \prime}$, respectively, and so $\widetilde{\Lambda}=\widetilde{\Lambda}^{\prime \prime}$.

To establish the claim that each vertex group $\Lambda(V)$ of $\Lambda$ is generated by the vertex groups of $\Lambda^{\prime \prime}$ that it contains, we will show that each edge group of $\Lambda$ for edges incident to $V$ is contained in a vertex group of $\Lambda^{\prime \prime}$ which is contained in $\Lambda(V)$. A generator of $\Lambda(V)$ which is not contained in any edge group incident to $V$ is an element of only that vertex group of $\Lambda$, but is also an element of some vertex group of $\Lambda^{\prime \prime}$ and that vertex group of $\Lambda^{\prime \prime}$ can only be contained in $\Lambda(V)$. Thus we will get that each generator of $\Lambda(V)$ is in a vertex group of $\Lambda^{\prime \prime}$ which is contained in $\Lambda(V)$.

Consider then an edge group $C$ of an edge incident to $V$ in $\Lambda$. Delete the edges $E_{1}, \ldots E_{n}$ of the underlying tree of $\Lambda$ that are incident to $V$ with the edge group $C$, leaving a connected component $T_{0}$ containing $V$, and connected components $T_{1}, \ldots, T_{n}$ with $T_{i}$ containing the vertex $V_{i}$ of $E_{i}$ opposite $V$ for each $i$. Then $W=A *_{C} B$ where $A$ is the group generated by the vertex groups of the tree $T_{0}$ and 
$B$ is the group generated by the vertex groups of the forest $T_{1}, \ldots, T_{n}$. Neither $A$ nor $B$ equals $C$ as $\Lambda$ is reduced. Each vertex group of $\Lambda^{\prime \prime}$ is contained in a unique vertex group of $\Lambda$ and so is contained in either $A$ or $B$ but not in both, since the intersection of $A$ and $B$ is the edge group $C$. There is at least one vertex group of $\Lambda^{\prime \prime}$ in each of $A$ and $B$. Hence there are adjacent vertices of $\Lambda^{\prime \prime}$ having vertex groups one in $A$ and one in $B$, whose intersection is the edge group of $\Lambda^{\prime \prime}$ for the edge between these vertices. But the intersection of these vertex groups of $\Lambda^{\prime \prime}$ is also contained in $C$. Since the edge groups of $\Lambda$ and $\Lambda^{\prime \prime}$ are visible subgroups conjugate to $\langle J\rangle$, we have that $C$ is the edge group of $\Lambda^{\prime \prime}$ for the edge between these vertices of $\Lambda^{\prime \prime}$. Hence $C$ is contained in a vertex group of $\Lambda^{\prime \prime}$ contained in $A$. If this vertex group of $\Lambda^{\prime \prime}$ is contained in a vertex group in $A$ other than $V$, then $C$ would be contained in the edge groups in a geodesic path between between $V$ and this other vertex in $T_{0}$. But the edge groups for edges incident to $V$ in $T_{0}$ are different conjugates of $\langle J\rangle$ than $C$, since we deleted all edges incident to $V$ having $C$ as edge group. As $C$ cannot be contained in a different conjugate of $\langle J\rangle$, instead the vertex group of $\Lambda^{\prime \prime}$ that is in $A$ and contains $C$ is actually contained in the vertex group $\Lambda(V)$ of $V$ in $\Lambda$. This completes the analysis of the claim and so completes the proof of the lemma.

We now finish the proof of Theorem 6.1. By Lemma 6.3, the vertex groups of $\Lambda^{\prime \prime}$ are in fact equal to the vertex groups of $\Lambda$. Finally we compare $\Lambda$ and $\Lambda^{\prime \prime}$ with $\Lambda^{\prime}$. Each vertex group $G$ of $\Lambda^{\prime \prime}$ is a subgroup of a conjugate of a vertex group $G^{\prime}$ of $\Lambda^{\prime}$ which is in turn a subgroup of a conjugate of a vertex group $H$ of $\Lambda$. But $G$ is a vertex group of $\Lambda$ and cannot be contained in a conjugate of another vertex group of $\Lambda$ (since again $\Lambda$ is reduced). Hence $G=H, G^{*} \preceq G^{\prime *} \preceq G^{*}$ so $G^{*}=G^{\prime *}$, and each vertex group of $\Lambda$ is conjugate to a vertex group of $\Lambda^{\prime}$. On the other hand, if $H^{\prime}$ is a vertex group of $\Lambda^{\prime}$ then $H^{\prime}$ is a subgroup of a conjugate of a vertex group $H$ of $\Lambda$. But $H$ is also a vertex group of $\Lambda^{\prime \prime}$, is contained in a conjugate of a vertex group $G^{\prime}$ of $\Lambda^{\prime}$, so $G^{\prime}=H^{\prime}$ is conjugate to $H$. Hence the vertex groups of $\Lambda^{\prime}$ correspond to conjugate vertex groups of $\Lambda$, as required for (3). This completes the proof of Theorem 6.1.

Lemma 6.4 If $\Lambda$ is a reduced visual graph of groups decomposition for a Coxeter system $(W, S)$ of finite rank such that the edge groups of $\Lambda$ are conjugates, then $\Lambda$ can be twisted resulting in a new generating set $S_{*}$ for $W$ and a reduced visual graph of groups decomposition $\Psi$ such that the edge groups of $\Psi$ are all equal.

Proof If not, take an example of a $\Lambda$ for $(W, S)$ and an edge group $E_{1}$, having a minimum number of edges labelled by groups different from $E_{1}$, which cannot be twisted to a $\Psi$ with equal edge groups. Then some vertex group $V$ contains incident edge groups $E_{1}$ and $E_{2}$ with $E_{1} \neq E_{2}$. Let $W_{1}$ be the group generated by the vertex 
groups of $\Lambda$ that are joined to $V$ by a geodesic path in the underlying tree of $\Lambda$ terminating in an edge incident to $V$ labelled by $E_{1}$ and let $W_{2}$ be the group generated by the rest of the vertex groups of $\Lambda$. Then we have a free product decomposition $W=W_{1} * E_{1} W_{2}$ with $E_{2} \subset V \subset W_{2}$. Let $d \in W$ be of minimal length, with respect to $S$, such that $E_{1}=d E_{2} d^{-1}$. By considering the normal form for $d$ with respect to the amalgamated product $W_{1} * E_{1} W_{2}$, we deduce that $d \in W_{2}$.

Suppose $E_{1}=\left\langle S_{0}\right\rangle, E_{2}=\left\langle\bar{S}_{0}\right\rangle, W_{1}=\left\langle S_{1}\right\rangle$, and $W_{2}=\left\langle S_{2}\right\rangle$ for $S_{0}, \bar{S}_{0}, S_{1}$, $S_{2} \subset S$. By Lemma 4.3 , we have $d \bar{S}_{0} d^{-1}=S_{0}$. Then twist the visual decomposition $\left.\left\langle S_{1}\right\rangle *{ }_{{ }_{0}}\right\rangle\left\langle S_{2}\right\rangle$ by conjugating $S_{2}$ by $d$, giving $S_{*}=S_{1} \cup d S_{2} d^{-1}$, and corresponding $\Lambda_{*}$ which is obtained from $\Lambda$ by conjugating by $d$ each vertex group of $\Lambda$ that is contained in $W_{2}$ and by conjugating by $d$ each edge group of $\Lambda$ whose incident vertex groups are contained in $W_{2}$. An edge labelled $E_{1}$ cannot have both incident vertex groups contained in $W_{2}$, since $S_{0}$ would have to be contained in each vertex and edge group in a geodesic path, in the underlying tree of $\Lambda$, between such an edge and an edge with label $E_{1}$ incident at $V$. Hence all the edges having label $E_{1}$ originally still have label $E_{1}$ in $\Lambda_{*}$. The edge incident to $V$ labelled $E_{2}$ in the original $\Lambda$ is conjugated to $d E_{2} d^{-1}=E_{1}$ and so we have at least one more edge labelled by $E_{1}$, and hence at least one fewer edge labelled by a group different from $E_{1}$. Thus $\Lambda_{*}$ contradicts the minimality of the number of edges labelled by groups different from a particular edge group assumed for $\Lambda$. Instead, twisting to reduce the number of edges labelled by a group different from a chosen edge group must eventually transform a given $\Lambda$ to a graph of groups $\Psi$ having all the same edge groups.

Lemma 6.5 Let $\Psi$ be a reduced graph of groups decomposition for a Coxeter system $(W, S)$ of finite rank such that all of the edge groups of $\Psi$ are equal, and let $\Psi_{0}^{\prime}$ be a similar decomposition for $\left(W, S^{\prime}\right)$ such that each vertex group is conjugate to a vertex group of $\Psi$ and the equal edge groups of $\Psi_{0}^{\prime}$ are conjugate to the edge groups of $\Psi$. Then by a sequence of twists applied to $\Psi_{0}^{\prime}$ there results a new set of Coxeter generators $S_{*}^{\prime}$ and corresponding visual graph of groups $\Psi^{\prime}$ such that the vertex groups of $\Psi^{\prime}$ are equal to those of $\Psi$ and the edge groups of $\Psi^{\prime}$ are all equal and equal to the edge groups of $\Psi$, and hence $\widetilde{\Psi}=\widetilde{\Psi}^{\prime}$.

Proof Let $\tilde{T}$ be the Bass-Serre tree for $\tilde{\Psi}$. Then each vertex group $V^{\prime}$ of $\Psi_{0}^{\prime}$ stabilizes a v-vertex of $\tilde{T}$, but stabilizes at most one v-vertex since $V^{\prime}$ cannot be a subgroup of a conjugate of an edge group of $\Psi_{0}^{\prime}$, and the same is true for any $\Psi_{*}^{\prime}$ resulting by twists conjugating vertex groups and preserving the same edge groups from $\Psi_{0}^{\prime}$. Let $T_{0}$ be the spanning tree for the v-vertices of $\widetilde{T}$ that are stabilized by a vertex group of such a $\Psi_{*}^{\prime}$ and take $\Psi_{*}^{\prime}$ so that $T_{0}$ has a minimal number of vertices. The smallest $T_{0}$ can be is one v-vertex for each vertex group of $\Psi_{*}^{\prime}$ plus one e-vertex, 
corresponding to the common edge group of $\tilde{\Psi}$, connected to each of the v-vertices of $T_{0}$. In this case, conjugating $\Psi_{*}^{\prime}$ carries $T_{0}$ to the standard transversal $T_{1}$ of $\widetilde{T}$ and so takes $\Psi_{*}^{\prime}$ to a $\Psi^{\prime}$ having the same vertex and edge groups as $\Psi$.

Suppose instead that $T_{0}$ has more than one e-vertex. Suppose further that some v-vertex $w V$ of $T_{0}$, for $V$ a vertex group of $\Psi$, stabilized by a vertex group $V^{\prime}=w V w^{-1}$ of $\Psi_{*}^{\prime}$ has more than one edge of $T_{0}$ incident at that vertex. Let $E$ be the common edge group of $\Psi$ so there are e-vertices $u E$ and $v E$ adjacent to $w V$, the edge group $E^{\prime}$ of $\Psi_{*}^{\prime}$ is $u E u^{-1}=v E v^{-1}$, and $u v^{-1} \in V^{\prime}$. Twist $\Psi_{*}^{\prime}$ to $\Psi_{* *}^{\prime}$ by conjugating each vertex group of $\Psi_{*}^{\prime}$ stabilizing a v-vertex of $T_{0}$ on the $v E$ side of $w V$ by the element $u v^{-1}$. Then $u v^{-1} E^{\prime} v u^{-1}=u E u^{-1}=E^{\prime}$, and so edge groups have not changed.

If $V_{2}^{\prime}$ is a vertex group of $\Psi_{*}^{\prime}$ stabilizing a v-vertex $w_{2} V_{2}$ on the $v E$ side of $w V$, then $u v^{-1} V_{2}^{\prime} v u^{-1}$ stabilizes $u v^{-1} w_{2} V_{2}$. If $p$ is a geodesic path from $w V$ to $w_{2} V_{2}$ in $T_{0}$, then translating $p$ by $u v^{-1}$ results in a path from $u v^{-1} w V=w V$ to $u v^{-1} w_{2} V_{2}$. Since the first edge in $p$ is $v E$, the first edge in the translated path is $u E$. We conclude that the spanning tree for the v-vertices stabilized by $\Psi_{* *}^{\prime}$ consists of the part of $T_{0}$ on the $v E$ side of $w V$ translated by $u v^{-1}$ together with the rest of $T_{0}$. Since the e-vertex $v E$ is carried to the e-vertex $u E$ in the new spanning tree, there are fewer vertices in the new spanning tree, contradicting the minimality of $T_{0}$ for $\Psi_{*}^{\prime}$.

Finally suppose that all the v-vertices of $T_{0}$ that are stabilized by a vertex group of $\Psi_{*}^{\prime}$ are the leaves of $T_{0}$ (the end points of $T_{0}$ ), and that $T_{0}$ has at least two e-vertices. Then $T_{0}$ has a v-vertex that is not stabilized by a vertex group of $\Psi_{*}^{\prime}$. Let $\widetilde{T}^{\prime}$ be the Bass-Serre tree of $\widetilde{\Psi}_{*}^{\prime}$. Let $\widetilde{T}_{*}^{\prime}$ be the result of replacing, equivariantly with respect to the action of $W$, each translate of the standard transversal $T_{1}^{\prime}$ of $\widetilde{T}^{\prime}$ by a copy of $T_{0}$ so that $T_{0}$ is attached by identifying each vertex of $T_{0}$ stabilized by a vertex group $V^{\prime}$ of $\Psi_{*}^{\prime}$ with the vertex $V^{\prime}$ of $T_{1}^{\prime}$ (this vertex remains labelled $V^{\prime}$ ). In particular, the e-vertices of $\widetilde{T}^{\prime}$ (those that are labelled by cosets of the edge group of $\Psi_{*}^{\prime}$ ) are replaced by copies of the level one core of $T_{0}$ (the tree $T_{0}$ minus its leaves and their adjoining edges). Then $W$ acts on the tree $\widetilde{T}_{*}^{\prime}$ translating the vertices labelled by cosets of vertex groups of $\Psi_{*}^{\prime}$ in the same way as in $\widetilde{\Psi}_{*}^{\prime}$.

Define a map $\tau: \widetilde{T}_{*}^{\prime} \rightarrow \widetilde{T}$ by mapping the vertex $V^{\prime}$ of the attached $T_{0}$ to the vertex $w V$ of $T_{0}$ when a vertex group $V^{\prime}$ of $\Psi_{*}^{\prime}$ stabilizes the vertex $w V$ in $T_{0}$, and by mapping the translates of $T_{0}$ in $\tilde{T}_{*}^{\prime}$ isomorphically to corresponding translates of $T_{0}$ in $\widetilde{T}$ so as to make $\tau$ respect the action of $W$. Then $\tau$ is locally injective, since the cosets $u v^{\prime} E^{\prime}$ of the edge group $E^{\prime}$ in a given v-vertex $u V^{\prime}$ of $\widetilde{T}^{\prime}$, which is also a vertex of $\widetilde{T}_{*}^{\prime}$, correspond to the cosets $u w v E$ of the edge group $E$ in the v-vertex $\tau\left(u V^{\prime}\right)=u w V$ of $\widetilde{T}$ under the correspondence $v^{\prime}=w v w^{-1}$, since $E^{\prime}=w E w^{-1}$ and $V^{\prime}=w V w^{-1}$. Hence $\tau$ is injective, since $\tau$ is a map of trees. But $T_{0}$ has an 
interior v-vertex $U=t V$, corresponding to a vertex group $V$ of $\Psi$, which is not stabilized by a vertex group of $\Psi_{*}^{\prime}$. Now $U=\tau\left(U^{\prime}\right)$ where $U^{\prime}$ is an interior v-vertex of the attached $T_{0}$ in $\widetilde{T}_{*}^{\prime}$. Let $w^{-1} V^{\prime}$ be the v-vertex of $\widetilde{T}^{\prime}$ stabilized by $V$. Then $w^{-1} V^{\prime} w=V$, and so $V^{\prime}=w V w^{-1}$. Hence the vertex group $V^{\prime}$ of $\Psi_{*}^{\prime}$ stabilizes the vertex $w V$ in $T_{0}$, and so $\tau\left(t w^{-1} V^{\prime}\right)=t w^{-1} w V=t V=U$. As $t w^{-1} V^{\prime} \neq U^{\prime}$ in $\widetilde{T}_{*}^{\prime}$, we have a contradiction to $\tau$ being injective.

Theorem 6.6 (Decomposition Matching Theorem) Let $W$ be a finitely generated Coxeter group with two sets of Coxeter generators $S$ and $S^{\prime}$, and suppose $W$ has a nontrivial splitting as $A *_{C} B$. Then there are sequences of twists applied to $(W, S)$ and $\left(W, S^{\prime}\right)$ giving rise to Coxeter systems $\left(W, S_{*}\right)$ and $\left(W, S_{*}^{\prime}\right)$, respectively, such that there exists a nontrivial reduced visual graph of groups decomposition $\Psi$ of $\left(W, S_{*}\right)$ and a nontrivial reduced visual graph of groups decomposition $\Psi^{\prime}$ of $\left(W, S_{*}^{\prime}\right)$ having the same graphs and the same vertex and edge groups and all edge groups equal and a subgroup of a conjugate of $C$.

Proof Let $\Lambda$ and $\Lambda^{\prime}$ be visual graph of groups decompositions of $(W, S)$ and $\left(W, S^{\prime}\right)$, respectively, as in Theorem 6.1. By Lemma 6.4, we can twist $\Lambda$ to get a visual decomposition $\Psi$ of $\left(W, S_{*}\right)$ with one edge group, and we can twist $\Lambda^{\prime}$ to get a visual decomposition $\Psi_{0}^{\prime}$ with one edge group. By Lemma 6.5, we can twist $\Psi_{0}^{\prime}$ to a visual decomposition $\Psi_{1}^{\prime}$ of $\left(W, S_{*}^{\prime}\right)$ having the same vertex and edge groups as $\Psi$, so $\widetilde{\Psi}=\widetilde{\Psi}^{\prime}$. Now $\Psi$ and $\Psi_{1}^{\prime}$ only differ by expansions and contractions rearranging the edge group attachments to vertex groups. So there is a visual decomposition $\Psi^{\prime}$ of $\left(W, S_{*}^{\prime}\right)$ with the same graph of groups structure as $\Psi$.

\section{The Simplex Matching Theorem}

In this section, we apply Theorem 2.1 and the Decomposition Matching Theorem to prove the Simplex Matching Theorem.

Lemma 7.1 Let $(W, S)$ be a finite irreducible Coxeter system whose type is different from $\mathbf{A}_{5}$, and let $\alpha$ be an automorphism of $W$. Then $\alpha$ can be written as a composition of two automorphisms, $\alpha=\beta \gamma$, such that $\beta$ is reflection preserving and $\gamma$ induces the identity on $[W, W]$.

Proof If ( $W, S)$ has type $\mathbf{A}_{n}$, for $n \neq 5$, then it is well know that every automorphism of $\mathbf{A}_{n}$ is inner, and so $\beta=\alpha$ and $\gamma$ is the identity. The remaining cases follow from Franzsen and Howlett [8, Theorem 31], since the automorphisms $\alpha_{\zeta}, \alpha_{\xi}, \alpha_{\chi}$ in Theorem 31 induce the identity on $[W, W]$ (since $[W, W]$ is the kernel of $\zeta, \xi$ and $\chi$ ), and the remaining automorphisms described in the generating sets are reflection preserving. 
Proposition 7.2 (Franzsen and Howlett [8, Proposition 32]) Let $(W, S)$ be a finite Coxeter system, and let $\alpha$ be an automorphism of $W$ that preserves reflections. Then $\alpha$ maps each visible subgroup of $(W, S)$ to a conjugate of a visible subgroup of the same type.

Lemma 7.3 Let $(W, S)$ be a finite irreducible Coxeter system whose type is different from $\mathbf{A}_{5}$, and let $\alpha$ be an automorphism of $W$. Let $A \subseteq S$ be irreducible with $|A|>1$. Then $A$ and $\operatorname{Pc}(\alpha([\langle A\rangle,\langle A\rangle]))$ have the same type.

Proof By Lemma 7.1, we can write $\alpha$ is a composition of two automorphisms, $\alpha=\beta \gamma$, such that $\beta$ is reflection preserving and $\gamma$ induces the identity on $[W, W]$. By Proposition 7.2, the exists $w \in W$ and $B \subseteq S$ of the same type as $A$ such that $\beta(\langle A\rangle)=w\langle B\rangle w^{-1}$. Then by Lemma 4.16, we have

$$
\begin{aligned}
\operatorname{Pc}(\alpha([\langle A\rangle,\langle A\rangle])) & =\operatorname{Pc}(\beta([\langle A\rangle,\langle A\rangle])) \\
& =\operatorname{Pc}\left(w[\langle B\rangle,\langle B\rangle] w^{-1}\right) \\
& =w \operatorname{Pc}([\langle B\rangle,\langle B\rangle]) w^{-1}=w\langle B\rangle w^{-1} .
\end{aligned}
$$

Thus $A$ and $\operatorname{Pc}(\alpha([\langle A\rangle,\langle A\rangle]))$ have the same type.

The next lemma follows from Lemma 7.3.

Lemma 7.4 Let $(W, S)$ and $\left(W^{\prime}, S^{\prime}\right)$ be finite irreducible Coxeter systems whose type is different from $\mathbf{A}_{5}$, and let $\phi: W \rightarrow W^{\prime}$ be an isomorphism. Let $A \subseteq S$ be irreducible with $|A|>1$. Then $A$ and $\operatorname{Pc}(\phi([\langle A\rangle,\langle A\rangle]))$ have the same type.

Lemma 7.5 Let $W$ be a finitely generated Coxeter group with two sets of Coxeter generators $S$ and $S^{\prime}$. Let $S_{1} \subseteq S$ and $S_{1}^{\prime} \subseteq S^{\prime}$, and suppose that $W_{1}=\left\langle S_{1}\right\rangle=\left\langle S_{1}^{\prime}\right\rangle$. If the basic subsets of $S$ and $S^{\prime}$ match isomorphically, then the basic subsets of $S_{1}$ and $S_{1}^{\prime}$ match isomorphically.

Proof On the contrary, suppose $B_{1}$ is a base of $\left(W_{1}, S_{1}\right)$ that matches nonisomorphically a base $B_{1}^{\prime}$ of $\left(W_{1}, S_{1}^{\prime}\right)$. Without loss of generality, we may assume that $\left|\left\langle B_{1}\right\rangle\right|>\left|\left\langle B_{1}^{\prime}\right\rangle\right|$. Then either $B_{1}$ is of type $\mathbf{C}_{2 q+1}$ and $B_{1}^{\prime}$ is of type $\mathbf{B}_{2 q+1}$ for some $q \geq 1$ or $B_{1}$ is of type $\mathbf{D}_{2}(4 q+2)$ and $B_{1}^{\prime}$ is of type $\mathbf{D}_{2}(2 q+1)$ for some $q \geq 1$. Let $B$ be the base of $(W, S)$ containing $B_{1}$ and let $B^{\prime}$ be the base of $\left(W, S^{\prime}\right)$ matching $B$. Then $B$ is not of type $\mathbf{A}_{5}$.

By the Basic Matching Theorem, there is an isomorphism $\phi:\langle B\rangle \rightarrow\left\langle B^{\prime}\right\rangle$ that restricts to conjugation on $[\langle B\rangle,\langle B\rangle]$ by an element $w$ of $W$. By Lemma 7.4, we have 
that $B_{1}$ and $\operatorname{Pc}\left(\phi\left(\left[\left\langle B_{1}\right\rangle,\left\langle B_{1}\right\rangle\right]\right)\right)$ have the same type. Let $B_{1}^{\prime \prime} \subseteq B^{\prime}$ be such that $\operatorname{Pc}\left(\phi\left(\left[\left\langle B_{1}\right\rangle,\left\langle B_{1}\right\rangle\right]\right)\right)$ is conjugate to $\left\langle B_{1}^{\prime \prime}\right\rangle$ in $\left\langle B^{\prime}\right\rangle$. Then $B_{1}$ and $B_{1}^{\prime \prime}$ have the same type.

By Proposition 4.14, the parabolic closure of $\phi\left(\left[\left\langle B_{1}\right\rangle,\left\langle B_{1}\right\rangle\right]\right)$ in $\left(\left\langle B^{\prime}\right\rangle, B^{\prime}\right)$ is the same as the parabolic closure of $\phi\left(\left[\left\langle B_{1}\right\rangle,\left\langle B_{1}\right\rangle\right]\right)$ in $\left(W, S^{\prime}\right)$. By the Basic Matching Theorem, there is an $w_{1} \in W_{1}$ such that

$$
\left[\left\langle B_{1}\right\rangle,\left\langle B_{1}\right\rangle\right]=w_{1}\left[\left\langle B_{1}^{\prime}\right\rangle,\left\langle B_{1}^{\prime}\right\rangle\right] w_{1}^{-1} .
$$

By Lemma 4.16 at the last step, we have

$$
\begin{aligned}
\operatorname{Pc}\left(\phi\left(\left[\left\langle B_{1}\right\rangle,\left\langle B_{1}\right\rangle\right]\right)\right) & =\operatorname{Pc}\left(w\left[\left\langle B_{1}\right\rangle,\left\langle B_{1}\right\rangle\right] w^{-1}\right) \\
& =w \operatorname{Pc}\left(\left[\left\langle B_{1}\right\rangle,\left\langle B_{1}\right\rangle\right]\right) w^{-1} \\
& =w \operatorname{Pc}\left(w_{1}\left[\left\langle B_{1}^{\prime}\right\rangle,\left\langle B_{1}^{\prime}\right\rangle\right] w_{1}^{-1}\right) w^{-1} \\
& =w w_{1} \operatorname{Pc}\left(\left[\left\langle B_{1}^{\prime}\right\rangle,\left\langle B_{1}^{\prime}\right\rangle\right]\right) w_{1}^{-1} w^{-1} \\
& =w w_{1}\left\langle B_{1}^{\prime}\right\rangle\left(w w_{1}\right)^{-1} .
\end{aligned}
$$

Hence $\left\langle B_{1}^{\prime \prime}\right\rangle$ and $\left\langle B_{1}^{\prime}\right\rangle$ are conjugate in $W$, and so $B_{1}^{\prime \prime}$ and $B_{1}^{\prime}$ have the same type. Therefore $B_{1}$ and $B_{1}^{\prime}$ have the same type, which is a contradiction.

The next lemma is known to experts; for a proof, see Paris [12].

Lemma 7.6 Let $W$ be a finitely generated Coxeter group with two complete Coxeter systems $(W, S)$ and $\left(W, S^{\prime}\right)$. Let

$$
(W, S)=\left(W_{0}, S_{0}\right) \times\left(W_{1}, S_{1}\right) \times \cdots \times\left(W_{k}, S_{k}\right)
$$

with $\left(W_{0}, S_{0}\right)$ finite and $\left(W_{i}, S_{i}\right)$ infinite and irreducible for each $i=1, \ldots, k$. Suppose

$$
\left(W, S^{\prime}\right)=\left(W_{0}^{\prime}, S_{0}^{\prime}\right) \times\left(W_{1}^{\prime}, S_{1}^{\prime}\right) \times \cdots \times\left(W_{\ell}^{\prime}, S_{\ell}^{\prime}\right)
$$

with $\left(W_{0}^{\prime}, S_{0}^{\prime}\right)$ finite and $\left(W_{j}^{\prime}, S_{j}^{\prime}\right)$ infinite and irreducible for each $j=1, \ldots, \ell$. Then $W_{0}=W_{0}^{\prime}$. Let $Z=Z\left(W_{0}\right)$. Then $k=\ell$ and after reindexing we have $Z W_{i}=Z W_{i}^{\prime}$ for each $i=1, \ldots, k$.

Theorem 7.7 (Simplex Matching Theorem) If $W$ is a finitely generated Coxeter group with two sets of Coxeter generators $S$ and $S^{\prime}$ whose basic subsets match isomorphically, then $(W, S)$ and $\left(W, S^{\prime}\right)$ have the same number of visible subgroups of each complete system isomorphism type. In particular, $|S|=\left|S^{\prime}\right|$, and the presentation diagrams of $(W, S)$ and $\left(W, S^{\prime}\right)$ have the same multiset of edge labels. 
Proof The proof is by induction on $|S|$. This is clear if $|S|=1$, so assume $|S|>1$ and the theorem is true for all Coxeter systems with fewer generators than $|S|$. Assume first that $(W, S)$ is complete. Then $\left(W, S^{\prime}\right)$ is complete by Proposition 4.21. Let

$$
(W, S)=\left(W_{1}, S_{1}\right) \times \cdots \times\left(W_{n}, S_{n}\right)
$$

be the factorization of $(W, S)$ into irreducible factors and suppose $\left(W_{i}, S_{i}\right)$ is finite if and only if $i \leq k$. Let

$$
\left(W, S^{\prime}\right)=\left(W_{1}^{\prime}, S_{1}^{\prime}\right) \times \cdots \times\left(W_{m}^{\prime}, S_{m}^{\prime}\right)
$$

be the factorization of $\left(W, S^{\prime}\right)$ into irreducible factors and suppose $\left(W_{i}, S_{i}^{\prime}\right)$ is finite if and only if $i \leq \ell$. By Lemma 7.6, we have

$$
\left(W_{1}, S_{1}\right) \times \cdots \times\left(W_{k}, S_{k}\right)=\left(W_{1}^{\prime}, S_{1}^{\prime}\right) \times \cdots \times\left(W_{\ell}^{\prime}, S_{\ell}^{\prime}\right) .
$$

By the Matching Theorem for systems of a finite Coxeter group, we can reindex so that $W_{i}$ is noncyclic if and only if $i \leq p$ and $W_{j}^{\prime}$ is noncyclic if and only if $j \leq p$ and $\left[W_{i}, W_{i}\right]=\left[W_{i}^{\prime}, W_{i}^{\prime}\right]$ for each $i \leq p$. By hypothesis, $\left(W_{i}, S_{i}\right) \cong\left(W_{i}^{\prime}, S_{i}^{\prime}\right)$ for each $i \leq p$. As the remaining finite factors have order 2, we have $k=\ell$ and $\left(W_{i}, S_{i}\right) \cong\left(W_{i}, S_{i}^{\prime}\right)$ for $p<i \leq k$.

By quotienting out the finite normal subgroup $\left(W_{1}, S_{1}\right) \times \cdots \times\left(W_{k}, S_{k}\right)$, we may assume that $W_{i}$ and $W_{j}^{\prime}$ are infinite for each $i$ and $j$. By Lemma 7.6, we have that $m=n$ and after reindexing $W_{i}=W_{i}^{\prime}$ for each $i$. Hence we may assume that $W$ is infinite and $(W, S)$ and $\left(W, S^{\prime}\right)$ are irreducible. By Theorem 2.1, we have that $(W, S) \cong\left(W, S^{\prime}\right)$. Thus in general $(W, S) \cong\left(W, S^{\prime}\right)$ when $(W, S)$ is complete.

Now assume $(W, S)$ is incomplete. Then there are $a, b$ in $S$ such that $m(a, b)=\infty$. Hence

$$
W=\langle S-\{a\}\rangle *\langle S-\{a, b\}\rangle\langle S-\{b\}\rangle
$$

is a nontrivial visual amalgamated decomposition. By the Decomposition Matching Theorem, there exist four nontrivial reduced visual graph of group decomposition of $W$, visual with respect to different sets of generators, a $\Lambda$ with respect to $S$, a $\Lambda^{\prime}$ with respect to $S^{\prime}$, a $\Psi$ with respect to another set of Coxeter generators $R$ of $W$, and a $\Psi^{\prime}$ with respect to another set of Coxeter generators $R^{\prime}$ of $W$ such that

(1) the edge groups of $\Lambda$ and $\Lambda^{\prime}$ are all conjugate and conjugate to a subgroup of $\langle S-\{a, b\}\rangle$

(2) there is a 1-1 correspondence between the vertices of $\Lambda$ and the vertices of $\Lambda^{\prime}$ such that each vertex group of $\Lambda$ is conjugate to the corresponding vertex group of $\Lambda^{\prime}$; 
(3) $\Psi$ is a twisted form of $\Lambda$ having all edge groups equal and conjugate to the edge groups of $\Lambda$, and having vertices in a 1-1 correspondence with those of $\Lambda$ such that each vertex group of $\Psi$ is conjugate to the corresponding vertex group of $\Lambda$, and $\Psi^{\prime}$ is similarly a twisted form of $\Lambda^{\prime}$; and

(4) $\Psi^{\prime}$ is the same graph of groups as $\Psi$ and differs from $\Psi$ only in being a visual graph of groups decomposition of $W$ with respect to a different set of Coxeter generators.

The Coxeter systems $(W, R)$ and $(W, S)$ are twist equivalent and so have the same number of visible subgroups of each complete system isomorphism type. Moreover $R$ and $S$ have isomorphically matching basic subsets. Likewise the Coxeter systems $\left(W, R^{\prime}\right)$ and $\left(W, S^{\prime}\right)$ have the same number of visible subgroups of each complete system isomorphism type, and $R^{\prime}$ and $S^{\prime}$ have isomorphically matching basic subsets. Let $\left\{\left(W_{i}, R_{i}\right)\right\}_{i=1}^{k}$ be the Coxeter systems of the vertex groups of $\Psi$, and let $\left(W_{0}, R_{0}\right)$ be the Coxeter system of the edge group of $\Psi$. Then $k \geq 2, R=\cup_{i=1}^{k} R_{i}$, and $\cap_{i=1}^{k} R_{i}=R_{0}$, and $R_{0} \neq R_{i}$ for each $i>0$, and $m(a, b)=\infty$ for each $a$ in $R_{i}-R_{0}$ and $b$ in $R_{j}-R_{0}$ with $i \neq j$. Let $\left\{\left(W_{i}^{\prime}, R_{i}^{\prime}\right)\right\}_{i=1}^{k}$ be the Coxeter systems of the vertex groups of $\Psi^{\prime}$ indexed so that $W_{i}^{\prime}=W_{i}$ for each $i$, and let $\left(W_{0}^{\prime}, R_{0}^{\prime}\right)$ be the Coxeter system of the edge group of $\Psi^{\prime}$. Then $W_{0}^{\prime}=W_{0}, R^{\prime}=\cup_{i=1}^{k} R_{i}^{\prime}$, and $\cap_{i=1}^{k} R_{i}^{\prime}=R_{0}^{\prime}$, and $R_{0}^{\prime} \neq R_{i}^{\prime}$ for each $i>0$, and $m\left(a^{\prime}, b^{\prime}\right)=\infty$ for each $a^{\prime}$ in $R_{i}^{\prime}-R_{0}^{\prime}$ and $b^{\prime}$ in $R_{j}^{\prime}-R_{0}^{\prime}$ with $i \neq j$. Moreover $R_{i}$ and $R_{i}^{\prime}$ have isomorphically matching basic subsets for each $i$ by Lemma 7.5.

Let $\mathcal{C}$ be a complete system isomorphism type and let $\mathcal{C}(S)$ be the number of visible subgroups of $(W, S)$ of isomorphism type $\mathcal{C}$. By the induction hypothesis, $\mathcal{C}\left(R_{i}\right)=$ $\mathcal{C}\left(R_{i}^{\prime}\right)$ for each $i$. Observe that

$$
\begin{aligned}
\mathcal{C}(S)=\mathcal{C}(R) & =\sum_{i=1}^{k} \mathcal{C}\left(R_{i}\right)-(k-1) \mathcal{C}\left(R_{0}\right) \\
& =\sum_{i=1}^{k} \mathcal{C}\left(R_{i}^{\prime}\right)-(k-1) \mathcal{C}\left(R_{0}^{\prime}\right)=\mathcal{C}\left(R^{\prime}\right)=\mathcal{C}\left(S^{\prime}\right),
\end{aligned}
$$

which completes the induction.

\section{Blowing up Coxeter systems}

Let $(W, S)$ be a Coxeter system of finite rank. In this section, we determine necessary and sufficient conditions on $(W, S)$ for a base $B$ of $(W, S)$ to match a base $B^{\prime}$ of $\left(W, S^{\prime}\right)$ with $|\langle B\rangle|>\left|\left\langle B^{\prime}\right\rangle\right|$. 
Lemma 8.1 Suppose $B$ is a base of $(W, S)$ of type $\mathbf{C}_{2 q+1}$ that matches a base $B^{\prime}$ of $\left(W, S^{\prime}\right)$ of type $\mathbf{B}_{2 q+1}$ for some $q \geq 1$. Let $a, b, c$ be the elements of $B$ such that $m(a, b)=4$ and $m(b, c)=3$, and let $A \subseteq S$ such that $a \in A$. If $\langle A\rangle$ is conjugate to $\left\langle A^{\prime}\right\rangle$ for some $A^{\prime} \subseteq S^{\prime}$, then $B \subseteq A$.

Proof Let $M \subseteq S$ be a maximal spherical simplex containing $B$. Then there is a unique maximal spherical simplex $M^{\prime} \subseteq S^{\prime}$ such that $\langle M\rangle$ is conjugate to $\left\langle M^{\prime}\right\rangle$ by Proposition 4.13. By conjugating $S^{\prime}$, we may assume that $\langle M\rangle=\left\langle M^{\prime}\right\rangle$. Then $M^{\prime}$ contains $B^{\prime}$ by the Basic Matching Theorem. Let $w$ be an element of $W$ such that $\langle A\rangle=w\left\langle A^{\prime}\right\rangle w^{-1}$. By Proposition 4.14, there is an element $x$ of $\left\langle M^{\prime}\right\rangle$ and a subset $C$ of $M^{\prime}$ such that

$$
\langle M \cap A\rangle=\langle M\rangle \cap\langle A\rangle=\left\langle M^{\prime}\right\rangle \cap w\left\langle A^{\prime}\right\rangle w^{-1}=x\langle C\rangle x^{-1} .
$$

Hence, we may assume that $W$ is finite by restricting to $\langle M\rangle$. Furthermore, by conjugating $S^{\prime}$, we may assume that $\langle A\rangle=\left\langle A^{\prime}\right\rangle$.

Let $D$ be a base of $(W, S)$ other than $B$. Then each element of $D$ commutes with each element of $B$, and so $\langle B\rangle$ injects into the quotient of $W$ by the commutator subgroup of $\langle D\rangle$. Hence, by Theorem 3.6, we may assume that $W$ is the direct product of $\langle B\rangle$ and copies of $\mathbf{A}_{1}$. The center $Z$ of $W$ is generated by $S-B$ and the longest element $z$ of $\langle B\rangle$. The center $Z$ is also generated by $S^{\prime}-B^{\prime}$. Let $K$ be the kernel of the homomorphism of $Z$ to $\langle z\rangle$ that maps $S-B$ to 1 and $z$ to $z$. Then $W / K$ is a Coxeter group. P-diagrams for $W / K$ are obtained from the $\mathrm{P}$-diagram of $(W, S)$ by removing the vertices $S-B$ and from the P-diagram of $\left(W, S^{\prime}\right)$ by removing the vertices in $\left(S^{\prime}-B^{\prime}\right) \cap K$ and identifying the remaining vertices of $S^{\prime}-B^{\prime}$ to a single vertex $z^{\prime}$. By passing to the quotient $W / K$, we may assume that $S=B$ and $S^{\prime}=B^{\prime} \cup\left\{z^{\prime}\right\}$ and $z^{\prime}$ commutes with each element of $B^{\prime}$. Then $z=z^{\prime}$, since $\langle z\rangle=Z(W)=\left\langle z^{\prime}\right\rangle$.

Now as $a \in A$ and $\langle A\rangle=\left\langle A^{\prime}\right\rangle$, we have that $a \in\left\langle A^{\prime}\right\rangle$. The element $a$ is represented by the matrix $\operatorname{diag}(1, \ldots, 1,-1)$ in $\mathbf{C}_{2 q+1}$. Observe that

$$
\operatorname{diag}(1, \ldots, 1,-1)=\operatorname{diag}(-1, \ldots,-1,-1) \operatorname{diag}(-1, \ldots,-1,1) .
$$

The matrix $-I$ represents $z$. The matrix $\operatorname{diag}(-1, \ldots,-1,1)$ is the longest element of $\mathbf{B}_{2 q+1}$ which is in $\left[\mathbf{C}_{2 q+1}, \mathbf{C}_{2 q+1}\right]$. Hence $\operatorname{diag}(-1, \ldots,-1,1)$ represents an element $\ell$ of $[\langle B\rangle,\langle B\rangle]$, with $a=\ell z$. As $[\langle B\rangle,\langle B\rangle]=\left[\left\langle B^{\prime}\right\rangle,\left\langle B^{\prime}\right\rangle\right]$, we have $\ell \in\left\langle B^{\prime}\right\rangle$. Hence every reduced word in the generators $S^{\prime}=B^{\prime} \cup\{z\}$ representing $a=\ell z$ involves $z$ by Bourbaki [1, Proposition 7]. Therefore $z \in A^{\prime}$, since $A^{\prime} \subseteq B^{\prime} \cup\{z\}$ and $a \in\left\langle A^{\prime}\right\rangle$. Hence $z \in\langle A\rangle$. As $z$ involves all the elements of $B$, we deduce that $B \subseteq A$. 
If $a \in S$, the neighborhood of $a$ in P-diagram of $(W, S)$ is defined to be the set $N(a)=\{s \in S: m(s, a)<\infty\}$.

If $A \subseteq S$, define $A^{\perp}=\{s \in S: m(s, a)=2$ for all $a \in A\}$.

Theorem 8.2 Suppose $B$ is a base of $(W, S)$ of type $\mathbf{C}_{2 q+1}$ that matches a base $B^{\prime}$ of $\left(W, S^{\prime}\right)$ of type $\mathbf{B}_{2 q+1}$ for some $q \geq 1$. Let $a, b, c$ be the elements of $B$ such that $m(a, b)=4$ and $m(b, c)=3$. Then $N(a)=B \cup B^{\perp}$.

Proof Let $s \in N(a)-B$, and let $A \subseteq S$ be a maximal spherical simplex containing $\{a, s\}$. Then there is a maximal spherical simplex $A^{\prime} \subseteq S^{\prime}$ such that $\langle A\rangle$ is conjugate to $\left\langle A^{\prime}\right\rangle$. Hence $B \subseteq A$ by Lemma 8.1. As $B$ is a base of $(\langle A\rangle, A)$, we deduce that $s \in B^{\perp}$.

Lemma 8.3 Let $w=s_{1} \cdots s_{n}$ be a reduced word in $(W, S)$ and let $s \in S$ such that $s \neq s_{i}$ for each $i=1, \ldots, n$. If $s w$ has finite order in $W$, then $m\left(s, s_{i}\right)<\infty$ for each $i=1, \ldots, n$.

Proof On the contrary, suppose $m\left(s, s_{i}\right)=\infty$ for some $i$. We may assume $S=$ $\left\{s, s_{1}, \ldots, s_{n}\right\}$. Then

$$
W=\left\langle s, s_{1}, \ldots, \widehat{s}_{i}, \ldots, s_{n}\right\rangle *\left\langle s_{1}, \ldots, \widehat{s}_{i}, \ldots, s_{n}\right\rangle\left\langle s_{1}, \ldots, s_{n}\right\rangle
$$

is a free product with amalgamation decomposition. Observe that $(s w)^{k}=s w s w \cdots s w$ is a normal form for $(s w)^{k}$ for each $k \geq 1$ with respect to the amalgamated product, and so $(s w)^{k} \neq 1$ for each $k \geq 1$.

Theorem 8.4 Let $B$ be a base of $(W, S)$ of type $\mathbf{C}_{2 q+1}$ for some $q \geq 1$, and let $a, b, c$ be the elements of $B$ such that $m(a, b)=4$ and $m(b, c)=3$. Suppose that $N(a)=B \cup B^{\perp}$. Let $d=a b a$, and let $z$ be the longest element of $\langle B\rangle$. Let $S^{\prime}=(S-\{a\}) \cup\{d, z\}$ and $B^{\prime}=(B-\{a\}) \cup\{d\}$. Then $S^{\prime}$ is a set of Coxeter generators for $W$ such that

(1) the set $B^{\prime}$ is a base of $\left(W, S^{\prime}\right)$ of type $\mathbf{B}_{2 q+1}$ that matches $B$,

(2) $\left(B^{\prime}\right)^{\perp}=B^{\perp} \cup\{z\}$, and $\{z\}$ is a component of $\left(B^{\prime}\right)^{\perp}$,

(3) $\quad N(d)=N(z)=B^{\prime} \cup\left(B^{\prime}\right)^{\perp}$ and

(4) the basic subsets of $S$ and $S^{\prime}$ are the same except for $B$ and $B^{\prime}$. 
Proof Consider the Coxeter presentation

$$
\left.W=\langle S|(s t)^{m(s, t)}: s, t \in S \text { and } m(s, t)<\infty\right\rangle .
$$

Now $\left(\left\langle B^{\prime}\right\rangle, B^{\prime}\right)$ is a finite Coxeter system of type $\mathbf{B}_{2 q+1}$. Let $\ell$ be the longest element of $\left(\left\langle B^{\prime}\right\rangle, B^{\prime}\right)$. Regard $\ell$ as a reduced word in the elements of $B^{\prime}$. Add generators $d$ and $z$ and relations $d=a b a$ and $z=a \ell$ to the above presentation for $W$. Now add the relators $(s t)^{m(s, t)}$ for $(s, t)$ in $\{d, z\} \times S^{\prime}$ or in $S^{\prime} \times\{d, z\}$ where $m(s, t)$ is the order of $s t$ in $W$ and $m(s, t)<\infty$. This includes all the relators of $\left(\left\langle B^{\prime}\right\rangle, B^{\prime}\right)$. As $\langle z\rangle$ is the center of $\langle B\rangle$, we have that $m(z, t)=2$ for all $t$ in $B^{\prime}$.

Next delete the generator $a$ and the relation $z=a \ell$ and replace $a$ by $z \ell$ in the remaining relations. As $z$ commutes with each element of $B^{\prime}$, we can replace the relation $d=z \ell b z \ell$ by the relation $d=\ell b \ell$.

The relators $(z \ell b)^{4}$ and $(b z \ell)^{4}$ can be replaced by $(\ell b)^{4}$ and $(b \ell)^{4}$ which in turn can be replaced by $(d b)^{2}$ and $(b d)^{2}$ using the relation $d=\ell b \ell$. The relators $(d b)^{2}$ and $(b d)^{2}$ are redundant and so we delete them. The relation $d=\ell b \ell$ is derivable from the relators of $\left(\left\langle B^{\prime}\right\rangle, B^{\prime}\right)$ and so we delete it. The relators $(z \ell s)^{2}$ and $(s z \ell)^{2}$ for $s \in B-\{a, b\}$ can be replaced by $(\ell s)^{2}$ and $(s \ell)^{2}$. The relators $(\ell s)^{2}$ and $(s \ell)^{2}$ are derivable from the relators of $\left(\left\langle B^{\prime}\right\rangle, B^{\prime}\right)$ and so we delete them.

Suppose $s \in N(a)-B$. Then $s \in B^{\perp}$ by hypothesis. Hence $m(s, t)=2$ for all $t \in B^{\prime} \cup\{z\}$. Now the relators $(z \ell s)^{2}$ and $(s z \ell)^{2}$ can be replaced by $(\ell s)^{2}$ and $(s \ell)^{2}$. The relators $(\ell s)^{2}$ and $(s \ell)^{2}$ are derivable from the relators $(s t)^{2}$ for $t \in B^{\prime}$ and the relation $\ell^{2}=1$. Hence we may delete the relators $(\ell s)^{2}$ and $(s \ell)^{2}$. This leaves the Coxeter presentation

$$
\left.W=\left\langle S^{\prime}\right|(s t)^{m(s, t)}: s, t \in S^{\prime} \text { and } m(s, t)<\infty\right\rangle .
$$

Thus $S^{\prime}$ is a set of Coxeter generators for $W$.

Statements 2 and 3 follow from Lemma 8.3 and the hypothesis that $N(a)=B \cup B^{\perp}$. The set $B^{\prime}$ is a base of $\left(W, S^{\prime}\right)$, since $N(d)=B^{\prime} \cup\left(B^{\prime}\right)^{\perp}$. The base $B$ matches the base $B^{\prime}$, since $[\langle B\rangle,\langle B\rangle]=\left[\left\langle B^{\prime}\right\rangle,\left\langle B^{\prime}\right\rangle\right]$. Statement 4 follows from Statements 1-3.

The next theorem follows from Theorem 8.2 and Theorem 8.4.

Theorem 8.5 Let $(W, S)$ be a Coxeter system of finite rank. Let $B$ be a base of $(W, S)$ of type $\mathbf{C}_{2 q+1}$ for some $q \geq 1$, and let $a, b, c$ be the elements of $B$ such that $m(a, b)=4$ and $m(b, c)=3$. Then $W$ has a set of Coxeter generators $S^{\prime}$ such that $B$ matches a base $B^{\prime}$ of $\left(W, S^{\prime}\right)$ of type $\mathbf{B}_{2 q+1}$ if and only if $N(a)=B \cup B^{\perp}$.

We now consider the dihedral case. 
Lemma 8.6 Suppose $B$ is a base of $(W, S)$ of type $\mathbf{D}_{2}(4 q+2)$ that matches a base $B^{\prime}$ of $\left(W, S^{\prime}\right)$ of type $\mathbf{D}_{2}(2 q+1)$. Then either $v=a$ or $v=b$ has the property that if $v \in A \subseteq S$ and $\langle A\rangle$ is conjugate to $\left\langle A^{\prime}\right\rangle$ for some $A^{\prime} \subseteq S^{\prime}$, then $B \subseteq A$.

Proof As in the proof of Lemma 8.1, we may assume that $\langle A\rangle=\left\langle A^{\prime}\right\rangle$ and reduce $W$ so that $S=B$ and $S^{\prime}=B^{\prime} \cup\{z\}$ where $\langle z\rangle$ is the center of $\langle B\rangle$. Now $a$ and $b$ are not both in $\left\langle B^{\prime}\right\rangle$. Choose $v$ so that $v$ is not in $\left\langle B^{\prime}\right\rangle$. Then every reduced $S^{\prime}$-word representing $v$ involves $z$. Now as $v \in A$, we have that $v \in\left\langle A^{\prime}\right\rangle$. Therefore $z \in A^{\prime}$. Hence $z \in\langle A\rangle$. Therefore $B \subseteq A$.

Theorem 8.7 Let $(W, S)$ be a Coxeter system of finite rank, and let $B=\{a, b\}$ be a base of $(W, S)$ of type $\mathbf{D}_{2}(4 q+2)$ for some $q \geq 1$. Then $W$ has a set of Coxeter generators $S^{\prime}$ such that $B$ matches a base $B^{\prime}$ of $\left(W, S^{\prime}\right)$ of type $\mathbf{D}_{2}(2 q+1)$ if and only if either $v=a$ or $v=b$ has the property that $N(v)=B \cup B^{\perp}$.

Proof Suppose that $W$ has a set of Coxeter generators $S^{\prime}$ such that $B$ matches a base $B^{\prime}$ of $\left(W, S^{\prime}\right)$ of type $\mathbf{D}_{2}(2 q+1)$. Let $v=a$ or $b$ as in Lemma 8.6. Suppose $s \in N(v)-B$. Let $A \subseteq S$ be a maximal spherical simplex containing $\{s, v\}$. Then there is a maximal spherical simplex $A^{\prime} \subseteq S^{\prime}$ such that $\langle A\rangle$ is conjugate to $\left\langle A^{\prime}\right\rangle$. Then $B \subseteq A$ by Lemma 8.6. As $B$ is a base of $(\langle A\rangle, A)$, we have $s \in B^{\perp}$. The converse follows from the next theorem.

Theorem 8.8 Let $B=\{a, b\}$ be a base of $(W, S)$ of type $\mathbf{D}_{2}(4 q+2)$ for some $q \geq 1$. Suppose that $N(a)=B \cup B^{\perp}$. Let $c=a b a$ and let $z$ be the longest element of $\langle B\rangle$. Let $S^{\prime}=(S-\{a\}) \cup\{c, z\}$ and $B^{\prime}=\{b, c\}$. Then $S^{\prime}$ is a set of Coxeter generators of $W$ such that

(1) the set $B^{\prime}$ is a base of $\left(W, S^{\prime}\right)$ of type $\mathbf{D}_{2}(2 q+1)$ that matches $B$,

(2) $\left(B^{\prime}\right)^{\perp}=B^{\perp} \cup\{z\}$, and $\{z\}$ is a component of $\left(B^{\prime}\right)^{\perp}$,

(3) $N(c)=N(z)=B^{\prime} \cup\left(B^{\prime}\right)^{\perp}$ and

(4) the basic subsets of $S$ and $S^{\prime}$ are the same except for $B$ and $B^{\prime}$.

Proof Consider the Coxeter presentation

$$
\left.W=\langle S|(s t)^{m(s, t)}: s, t \in S \text { and } m(s, t)<\infty\right\rangle .
$$

Now $\left(\left\langle B^{\prime}\right\rangle, B^{\prime}\right)$ is a finite Coxeter system of type $\mathbf{D}_{2}(2 q+1)$. Let $\ell$ be the longest element of $\left(\left\langle B^{\prime}\right\rangle, B^{\prime}\right)$. Regard $\ell$ as the reduced word $(b c)^{q} b$ in the elements of $B^{\prime}$. Add generators $c$ and $z$ and relations $c=a b a$ and $z=a \ell$ to the above presentation for $W$. Now add the relators $(s t)^{m(s, t)}$ for $(s, t)$ in $\{c, z\} \times S^{\prime}$ or in $S^{\prime} \times\{c, z\}$ where 
$m(s, t)$ is the order of $s t$ in $W$ and $m(s, t)<\infty$. This includes all the relators of $\left(\left\langle B^{\prime}\right\rangle, B^{\prime}\right)$. As $\langle z\rangle$ is the center of $\langle B\rangle$, we have that $m(z, b)=m(z, c)=2$.

Next delete the generator $a$ and the relation $z=a \ell$ and replace $a$ by $z \ell$ in the remaining relations. As $z$ commutes with each element of $B^{\prime}$, we can replace the relation $c=z \ell b z \ell$ by the relation $c=\ell b \ell$.

The relators $(z \ell b)^{2(2 q+1)}$ and $(b z \ell)^{2(2 q+1)}$ can be replaced by $(\ell b)^{2(2 q+1)}$ and $(b \ell)^{2(2 q+1)}$ which in turn can be replaced by $(c b)^{2 q+1}$ and $(b c)^{2 q+1}$ using the relation $c=\ell b \ell$. The relators $(c b)^{2 q+1}$ and $(b c)^{2 q+1}$ are redundant and so we delete them. The relation $c=\ell b \ell$ is derivable from the relators of $\left(\left\langle B^{\prime}\right\rangle, B^{\prime}\right)$ and so we delete it.

Suppose $s \in N(a)-B$. Then $s \in B^{\perp}$ by hypothesis. Hence $m(s, t)=2$ for $t \in\{b, c, z\}$. Now the relators $(z \ell s)^{2}$ and $(s z \ell)^{2}$ can be replaced by $(\ell s)^{2}$ and $(s \ell)^{2}$. The relators $(\ell s)^{2}$ and $(s \ell)^{2}$ are derivable from the relators $(s t)^{2}$ for $t \in B^{\prime}$ and the relation $\ell^{2}=1$. Hence we may delete the relators $(\ell s)^{2}$ and $(s \ell)^{2}$. This leaves the Coxeter presentation

$$
\left.W=\left\langle S^{\prime}\right|(s t)^{m(s, t)}: s, t \in S^{\prime} \text { and } m(s, t)<\infty\right\rangle .
$$

Thus $S^{\prime}$ is a set of Coxeter generators for $W$.

Statements 2 and 3 follow from Lemma 8.3 and the hypothesis that $N(a)=B \cup B^{\perp}$. The set $B^{\prime}$ is a base of $\left(W, S^{\prime}\right)$, since $N(d)=B^{\prime} \cup\left(B^{\prime}\right)^{\perp}$. The base $B$ matches the base $B^{\prime}$, since $[\langle B\rangle,\langle B\rangle]=\left[\left\langle B^{\prime}\right\rangle,\left\langle B^{\prime}\right\rangle\right]$. Statement 4 follows from Statements 1-3.

\section{The maximum rank of a Coxeter group}

Let $(W, S)$ be a Coxeter system of finite rank. We say that $(W, S)$ can be blown up along a base $B$ if $(W, S)$ and $B$ satisfy the hypothesis of either Theorem 8.4 or Theorem 8.8. If $(W, S)$ can be blown up along a base $B$, then we can blow up $(W, S)$ to a Coxeter system $\left(W, S^{\prime}\right)$ as in the statement of Theorem 8.4 or Theorem 8.8 such that $\left|S^{\prime}\right|=|S|+1$, the base $B$ matches a base $B^{\prime}$ of $\left(W, S^{\prime}\right)$ with $|\langle B\rangle|>\left|\left\langle B^{\prime}\right\rangle\right|$, and $S$ and $S^{\prime}$ have the same basic subsets except for $B$ and $B^{\prime}$. We say that $\left(W, S^{\prime}\right)$ is obtained by blowing up $(W, S)$ along the base $B$. The system $\left(W, S^{\prime}\right)$ is also called an elementary reduction of $(W, S)$ by Mühlherr [11].

By the process of blowing up along a base, we can effectively construct a sequence $S=S^{(0)}, S^{(1)}, \ldots, S^{(\ell)}$ of Coxeter generators of $W$ such that $S^{(i+1)}$ is obtained by blowing up $\left(W, S^{(i)}\right)$ along a base for each $i=0, \ldots, \ell-1$, and $\left(W, S^{(\ell)}\right)$ cannot be blown up along a base. The sequence terminates since the sum of the orders of the basic subgroups decreases at each step of the sequence. By the next theorem, the system $\left(W, S^{(\ell)}\right)$ has maximum rank over all Coxeter systems for $W$. 
Theorem 9.1 (Maximum Rank Theorem) Let $(W, S)$ be a Coxeter system of finite rank. Then the following are equivalent:

(1) we have $|S| \geq\left|S^{\prime}\right|$ for every set of Coxeter generators $S^{\prime}$ of $W$,

(2) each base of $(W, S)$ matches a base $B^{\prime}$ of $\left(W, S^{\prime}\right)$ with $|\langle B\rangle| \leq\left|\left\langle B^{\prime}\right\rangle\right|$ for every set of Coxeter generators $S^{\prime}$ of $W$ and

(3) the system $(W, S)$ cannot be blown up along a base.

Proof Suppose that $|S| \geq\left|S^{\prime}\right|$ for every set of Coxeter generators $S^{\prime}$ of $W$ and on the contrary, a base $B$ of $(W, S)$ matches a base $B^{\prime}$ of $\left(W, S^{\prime}\right)$ with $|\langle B\rangle|>\left|\left\langle B^{\prime}\right\rangle\right|$. By the Basic Matching Theorem either $B$ is of type $\mathbf{C}_{2 q+1}$ and $B^{\prime}$ is of type $\mathbf{B}_{2 q+1}$ for some $q \geq 1$ or $B$ is of type $\mathbf{D}_{2}(4 q+2)$ and $B^{\prime}$ is of type $\mathbf{D}_{2}(2 q+1)$ for some $q \geq 1$. By Theorem 8.5 and Theorem 8.7 , we have that $(W, S)$ and $B$ satisfy the hypothesis of Theorem 8.4 or Theorem 8.8 . Therefore $(W, S)$ can be blown up along $B$ to obtain a system $\left(W, S^{\prime}\right)$ with $\left|S^{\prime}\right|=|S|+1$ contrary to the maximality of $|S|$. Therefore (1) implies (2).

Suppose that each base $B$ of $(W, S)$ matches a base $B^{\prime}$ of $\left(W, S^{\prime}\right)$ with $|\langle B\rangle| \leq\left|\left\langle B^{\prime}\right\rangle\right|$ for every set of Coxeter generators $S^{\prime}$ of $W$. If $|\langle B\rangle|=\left|\left\langle B^{\prime}\right\rangle\right|$ for every base $B$ of $(W, S)$, then $S$ and $S^{\prime}$ have isomorphically matching basic subsets by the Basic Matching Theorem, and so $|S|=\left|S^{\prime}\right|$ by the Simplex Matching Theorem.

Suppose a base $B$ of $(W, S)$ matches a base $B^{\prime}$ of $\left(W, S^{\prime}\right)$ with $|\langle B\rangle|<\left|\left\langle B^{\prime}\right\rangle\right|$. By the Basic Matching Theorem either $B^{\prime}$ is of type $\mathbf{C}_{2 q+1}$ and $B$ is of type $\mathbf{B}_{2 q+1}$ for some $q \geq 1$ or $B^{\prime}$ is of type $\mathbf{D}_{2}(4 q+2)$ and $B$ is of type $\mathbf{D}_{2}(2 q+1)$ for some $q \geq 1$. By Theorem 8.5 and Theorem 8.7 , we have that $\left(W, S^{\prime}\right)$ and $B^{\prime}$ satisfy the hypothesis of Theorem 8.4 or Theorem 8.8 , and so $\left(W, S^{\prime}\right)$ can be blown up along $B^{\prime}$ to obtain a system $\left(W, S^{\prime \prime}\right)$ with $\left|S^{\prime \prime}\right|=\left|S^{\prime}\right|+1$ such that $B^{\prime}$ matches a base $B^{\prime \prime}$ of $\left(W, S^{\prime \prime}\right)$ with $\left|\left\langle B^{\prime}\right\rangle\right|>\left|\left\langle B^{\prime \prime}\right\rangle\right|=|\langle B\rangle|$, and $S^{\prime}$ and $S^{\prime \prime}$ have the same basic subsets except for $B^{\prime}$ and $B^{\prime \prime}$.

If $S$ and $S^{\prime \prime}$ do not have isomorphically matching basic subsets, we can blow up $\left(W, S^{\prime \prime}\right)$ along a base. Continuing in this way, we obtain a sequence of Coxeter generators $S^{\prime}=S^{(1)}, \ldots, S^{(\ell)}$ of $W$ such that $S^{(i+1)}$ is obtained by blowing up $\left(W, S^{(i)}\right)$ along a base for each $i=1, \ldots, \ell-1$, and $S$ and $S^{(\ell)}$ have isomorphically matching basic subsets. In particular, $\left|S^{(i+1)}\right|=\left|S^{(i)}\right|+1$ for each $i=1, \ldots, \ell-1$. By the Simplex Matching Theorem, $|S|=\left|S^{(\ell)}\right|$, and so $|S|>\left|S^{\prime}\right|$. Thus (2) implies (1).

Finally (2) and (3) are equivalent by the Basic Matching Theorem and Theorem 8.5 and Theorem 8.7 . 
Theorem 9.2 (Simplex Matching Theorem for maximum rank systems) If $W$ is a finitely generated Coxeter group and $S$ and $S^{\prime}$ are Coxeter generators of $W$ of maximum rank, then $(W, S)$ and $\left(W, S^{\prime}\right)$ have the same number of visible subgroups of each complete system isomorphism type.

Proof By the Maximum Rank Theorem, each base $B$ of $(W, S)$ matches a base $B^{\prime}$ of $\left(W, S^{\prime}\right)$ with $|\langle B\rangle|=\left|\left\langle B^{\prime}\right\rangle\right|$. Therefore $S$ and $S^{\prime}$ have isomorphically matching basic subsets by the Basic Matching Theorem. Hence $(W, S)$ and $\left(W, S^{\prime}\right)$ have the same number of visible subgroups of each complete system isomorphism type by the Simplex Matching Theorem.

\section{References}

[1] N Bourbaki, Éléments de mathématique Fasc. XXXIV: Groupes et algèbres de Lie, Chapitres IV-VI, Actualités Scientifiques et Industrielles 1337, Hermann, Paris (1968) MR0240238

[2] N Brady, J P McCammond, B Mühlherr, W D Neumann, Rigidity of Coxeter groups and Artin groups, Geom. Dedicata 94 (2002) 91-109 MR1950875

[3] B Brink, R B Howlett, Normalizers of parabolic subgroups in Coxeter groups, Invent. Math. 136 (1999) 323-351 MR1688445

[4] P-E Caprace, B Mühlherr, Reflection rigidity of 2-spherical Coxeter groups, Proc. London Math. Soc. 94 (2007) 520-542

[5] HSM Coxeter, The complete enumeration of finite groups of the form $R_{i}^{2}=$ $\left(R_{i} R_{j}\right)^{k_{i j}}=1$, J. London Math. Soc. 10 (1935) 21-25

[6] HS M Coxeter, Regular polytopes, third edition, Dover Publications, New York (1973) MR0370327

[7] V V Deodhar, On the root system of a Coxeter group, Comm. Algebra 10 (1982) 611-630 MR647210

[8] W N Franzsen, R B Howlett, Automorphisms of nearly finite Coxeter groups, Adv. Geom. 3 (2003) 301-338 MR1997410

[9] W N Franzsen, R B Howlett, B Mühlherr, Reflections in abstract Coxeter groups, Comment. Math. Helv. 81 (2006) 665-697 MR2250859

[10] M Mihalik, S Tschantz, Visual decompositions of Coxeter groups (2007) arXiv: math. GR/0703439v1

[11] B Mühlherr, The isomorphism problem for Coxeter groups, from: "The Coxeter legacy", Amer. Math. Soc., Providence, RI (2006) 1-15 MR2209018

[12] L Paris, Irreducible Coxeter groups, Internat. J. Algebra Comput. (to appear) arXiv: math. GR/0412214v1 
[13] L Solomon, A Mackey formula in the group ring of a Coxeter group, J. Algebra 41 (1976) 255-264 MR0444756

[14] M Suzuki, Group theory I, Grundlehren der Mathematischen Wissenschaften 247, Springer, Berlin (1982) MR648772

Mathematics Department, Vanderbilt University

Nashville TN 37240, USA

michael.l.mihalik@vanderbilt.edu, j.g.ratcliffe@vanderbilt.edu, steven.tschantz@vanderbilt.edu

Received: 31 March 2006

Algebraic 83 Geometric Topology, Volume 7 (2007) 